\title{
Stereochemical Studies of Sialic Acid Derivatives by Vibrational Circular Dichroism
}

\author{
Atsufumi Nakahashi, Tohru Taniguchi, Nobuaki Miura, and Kenji Monde*
}

Graduate School of Advanced Life Science, Frontier Research Center for Post-Genome Science and Technology, Hokkaido University, Kita-ku, Sapporo 001-0021, Japan

E-mail: kmonde@glyco.sci.hokudai.ac.jp

1. Contents.

2. General experimental details.

2-7. Synthetic procedures and spectral data of $8 \mathbf{a}, 8 \mathbf{b}, 9 \mathbf{a}, 9 \mathbf{b}, 10 \mathbf{a}$, and $10 \mathbf{b}$.

8-19. ${ }^{1} \mathrm{H},{ }^{13} \mathrm{C}$ NMR, ${ }^{1} \mathrm{H},{ }^{1} \mathrm{H}-\mathrm{COSY}, \mathrm{HMQC}$, and HMBC spectra of 8a, 8b, 9a, 9b, 10a, and $10 b$. 


\section{General Procedures.}

${ }^{1} \mathrm{H}$ NMR (600 MHz) and ${ }^{13} \mathrm{C}$ NMR $(150 \mathrm{MHz}$ or $125 \mathrm{MHz})$ spectra were recorded on a Bruker Avance spectrometer. Chemical shift values are reported in $\delta(\mathrm{ppm})$ values, and coupling constant values $(J)$ are in Hertz $(\mathrm{Hz})$. The following abbreviations were used for signal multiplicities: $\mathrm{s}=$ singlet; $\mathrm{d}=$ doublet; $\mathrm{t}=$ triplet; $\mathrm{m}=$ multiplet. FAB-MS was obtained on a JEOL JMS-HX110 at Center for Instrumental Analysis, Hokkaido University. Optical rotation was measured on a Perkin-Elmer 343 polarimeter using a 1 $\mathrm{cm}$ optical cell under an ambient temperature. Analytical TLC was performed on $0.2 \mathrm{~mm}$ silica gel plates (Merck $60 \mathrm{~F}-254$ ). $\mathrm{SiO}_{2}$ gel column chromatography was carried out using silica gel (Kanto $60 \mathrm{~N}, 40-50 \mu \mathrm{m}$ ) with air flashing or a Yamazen pump 700E. HPLC purification was conducted on a Shimadzu LC-6A liquid chromatograph instrument equipped with a Shimadzu SPD-6AV UV-Vis spectrophotomeric detector using a YMC-Pack SIL column $(2 \mathrm{~cm} \phi$ x $25 \mathrm{~cm})$. Reverse phase HPLC was performed using a HITACHI L-6200 HPLC system equipped with an Inertsil-ODS column (10 mm x $250 \mathrm{~mm}$, GL Scinences Inc.) and HITACHI L-7405 UV detector. $N$-Acetylneuraminic acid (2) and methyl $\beta$-D-galactopyranoside were purchased from Nacalai Tesque Inc. and Tokyo Kasei Kogyo Co. Ltd., respectively.

VCD spectroscopy. VCD spectra were measured on a Bomem/BioTools Chiralir spectrometer at a resolution of $8 \mathrm{~cm}^{-1}$ under an ambient temperature. All VCD spectra are the average of three blocks of 4500 scans. Samples were dissolved in DMSO- $d_{6}$ and then placed in a $71 \mu \mathrm{m} \mathrm{BaF} 2$ cell (SL-4, International Crystal Laboratories, Inc.) or a $100 \mu \mathrm{m}$ $\mathrm{CaF}_{2}$ cell (KIII $64744 \mathrm{JASCO}$ ). The concentrations were as follows: 5a, 5b: $0.18 \mathrm{M}$, and 10a, 10b: $0.16 \mathrm{M}$. The IR and VCD spectra were corrected by a solvent spectrum obtained at the same experimental conditions, and presented in molar absorptivity $\varepsilon$ (L/molcm).

\section{Synthesis of [Methyl 5-acetamido-4,7,8,9-tetra- $O$-acetyl-3,5-dideoxy- $\beta$-D-glycero $-\alpha / \beta$-D-galacto-2-nonulopyranosonate]-(2 $\rightarrow 6)$-methyl}

\section{2,3,4-tri- $\boldsymbol{O}$-benzyl- $\beta$-D-galacto -pyranoside (8a, 8b).}

Compounds 6 (3.00 g, $5.88 \mathrm{mmol})$ and 7 (3.18 g, $6.85 \mathrm{mmol}, 1.2 \mathrm{eq})$ were azeotroped with dry toluene two times, then evaporated in vacuo for $3 \mathrm{~h}$. A mixture of $\mathbf{6}, \mathbf{7}$, and $4 \mathrm{~A}$ molecular sieves in dry toluene $(100 \mathrm{~mL})$ was stirred at room temperature under nitrogen for $10 \mathrm{~min}$, then cooled to $-20^{\circ} \mathrm{C}$. AgOTf (2.20 g, $\left.8.56 \mathrm{mmol}, 1.5 \mathrm{eq}\right)$ dissolved in dry toluene $(30 \mathrm{~mL})$ was added to the mixture. After stirring at $-20^{\circ} \mathrm{C}$ in the dark for $12 \mathrm{~h}$, the mixture was warmed up to room temperature. The resulting mixture was filtered, concentrated, diluted with ethyl acetate $(250 \mathrm{~mL})$, and washed with water $(2 \times 250 \mathrm{~mL})$. 
The organic layer was washed again with brine $(250 \mathrm{~mL})$, dried over $\mathrm{MgSO}_{4}$, and concentrated. The residue was submitted to $\mathrm{SiO}_{2}$ gel column chromatography (toluene:ethyl acetate $=1: 2$ ), and further purified by flash column chromatography (chloroform:methanol $=100: 1)$ to give $\mathbf{8 a}(\alpha$ isomer) and $\mathbf{8 b}(\beta$ isomer) as amorphous solids in each $20 \%$ yield.

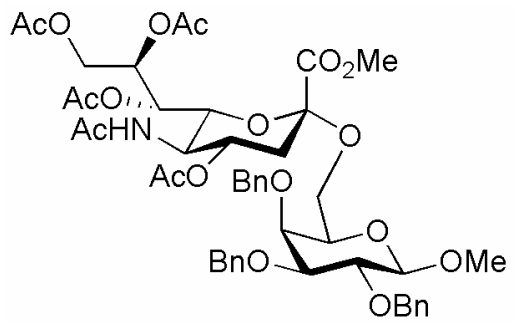

[Methyl 5-acetamido-4,7,8,9-tetra- $O$-acetyl-3,5-dideoxy- $\beta$-D-glycero - $\alpha$-D-galacto-2-nonulopyranosonate]-(2 $\rightarrow 6)$-methyl 2,3,4-tri- $O$-benzyl- $\beta$-D-galacto -pyranoside (8a).

$[\alpha]_{\mathrm{D}} 0.0^{\circ}\left(c 0.2, \mathrm{CHCl}_{3}\right) ;{ }^{1} \mathrm{H}$ NMR $\left(600 \mathrm{MHz}, \mathrm{CDCl}_{3}\right): \delta$ 7.38-7.21 (15H, m, ArH), 5.36 $(1 \mathrm{H}, \mathrm{m}, \mathrm{NeuAc}-\mathrm{H}-8), 5.32(1 \mathrm{H}, \mathrm{d}, J=8.0 \mathrm{~Hz}, \mathrm{NeuAc}-\mathrm{H}-7), 5.19(1 \mathrm{H}, \mathrm{d}, J=4.9 \mathrm{~Hz}, \mathrm{~N}-\mathrm{H})$, $4.96\left(1 \mathrm{H}, \mathrm{d}, J=11.5 \mathrm{~Hz}, \mathrm{PhCH}_{2}\right), 4.89\left(1 \mathrm{H}, \mathrm{d}, J=10.9 \mathrm{~Hz}, \mathrm{PhCH}_{2}\right), 4.85(1 \mathrm{H}, \mathrm{m}$, NeuAc-H-4), 4.77-4.69 (3H, m, $\left.\mathrm{PhCH}_{2}\right), 4.65\left(1 \mathrm{H}, \mathrm{d}, J=11.5 \mathrm{~Hz}, \mathrm{PhCH}_{2}\right), 4.33-4.29(2 \mathrm{H}$, m, NeuAc-H-9a and Gal-H-1), 4.11-4.04 (3H, m, NeuAc-H-5, NeuAc-H-6 and NeuAc-H-9b), 3.90 (1H, d, $J=2.8$ Hz, Gal-H-4), 3.88 (1H, dd, $J=5.4,8.7$ Hz, Gal-H-6a), 3.79 (1H, dd, $J=7.8,9.6 \mathrm{~Hz}, \mathrm{Gal}-\mathrm{H}-2), 3.61$ (3H, s, $\left.\mathrm{COOCH}_{3}\right), 3.60-3.50$ (6H, m, OMe, Gal-H-3, Gal-H-5 and Gal-H-6b), 2.59 (1H, dd, $J=4.7,12.8$ Hz, NeuAc-H-3eq), 2.13, 2.11, 2.03, 2.02 (3H each, s each, OAc), 1.95 (1H, dd, $J=12.6,12.7 \mathrm{~Hz}$, NeuAc-H-3ax), $1.88(3 \mathrm{H}, \mathrm{s}, \mathrm{NAc}) ;{ }^{13} \mathrm{C}$ NMR $\left(125 \mathrm{MHz}, \mathrm{CDCl}_{3}\right): \delta 170.9,170.6,170.2,170.1,169.8$, 168.0, 139.0, 138.9, 138.6, 128.3, 128.3, 128.1, 128.1, 127.7, 127.5, 127.5, 127.5, 127.2, 105.0, 98.8, 82.1, 79.6, 75.1, 74.3, 73.4, 73.0, 72.7, 72.6, 69.0, 68.8, 67.5, 62.8, 62.3, 57.1,

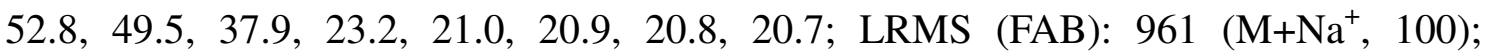
HRMS(FAB) Calcd for $\mathrm{C}_{48} \mathrm{H}_{59} \mathrm{NO}_{18} \mathrm{Na}\left(\mathrm{M}+\mathrm{Na}^{+}\right)$960.3630, found 960.3639.

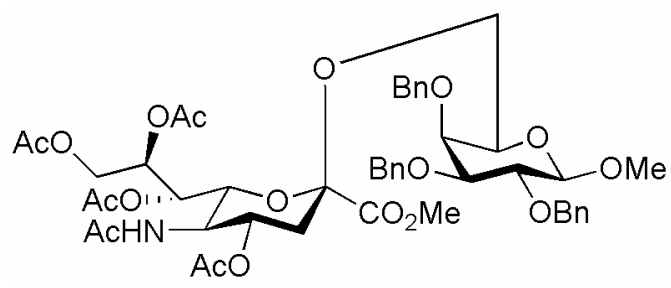

[Methyl 5-acetamido-4,7,8,9-tetra- $O$-acetyl-3,5-dideoxy- $\beta$-D-glycero - $\beta$-D-galacto-2-nonulopyranosonate]- $(2 \rightarrow 6)$-methyl $2,3,4$-tri- $O$-benzyl- $\beta$-D-galacto -pyranoside (8b). 
$[\alpha]_{\mathrm{D}}-20.0^{\circ}\left(c 0.5, \mathrm{CHCl}_{3}\right) ;{ }^{1} \mathrm{H} \mathrm{NMR}\left(600 \mathrm{MHz}, \mathrm{CDCl}_{3}\right): \oint$ 7.53-7.26 (15H, m, ArH), 5.46, $\left(1 \mathrm{H}, \mathrm{d}, J=13.5 \mathrm{~Hz}, \mathrm{PhCH}_{2}\right), 5.21(1 \mathrm{H}, \mathrm{m}, \mathrm{NeuAc}-\mathrm{H}-8), 5.10(1 \mathrm{H}, \mathrm{dd}, J=2.4,7.0 \mathrm{~Hz}$, NeuAc-H-7), 4.90-4.74 (5H, m, NeuAc-H-4 and $\left.\mathrm{PhCH}_{2}\right), 4.73(1 \mathrm{H}, \mathrm{d}, J=10.9 \mathrm{~Hz}$, $\left.\mathrm{PhCH}_{2}\right), 4.56(1 \mathrm{H}, \mathrm{dd}, J=2.5,12.5 \mathrm{~Hz}, \mathrm{NeuAc}-\mathrm{H}-9 \mathrm{a}), 4.30$ (1H, d, $\left.J=7.6 \mathrm{~Hz}, \mathrm{Gal}-\mathrm{H}-1\right)$, 4.09 (1H, d, $J=2.4$ Hz, Gal-H-4), 4.07-3.97 (2H, m, NeuAc-H-5 and NeuAc-H-9b), 3.81 $\left(3 \mathrm{H}, \mathrm{s}, \mathrm{COOCH}_{3}\right), 3.80-3.74(2 \mathrm{H}, \mathrm{m}, \mathrm{N}-\mathrm{H}$ and Gal-H-2), 3.69-3.59 (3H, m, Gal-H-3, Gal-H-6a and Gal-H-6b), 3.59-3.56 (4H, m, Gal-H-5 and OMe), 3.51 (1H, dd, $J=2.4$, 10.6 Hz, NeuAc-H-6), 2.27 (1H, dd, $J=4.9,12.8 \mathrm{~Hz}$, NeuAc-H-3eq), 2.24 (3H, s, OAc), 2.07 (6H, s, OAc), 2.00 (3H, s, OAc), 1.86 (1H, dd, $J=11.7,12.7 \mathrm{~Hz}$, NeuAc-H-3ax), $1.71(3 \mathrm{H}, \mathrm{s}, \mathrm{NAc}) ;{ }^{13} \mathrm{C}$ NMR $\left(125 \mathrm{MHz}, \mathrm{CDCl}_{3}\right): \delta 170.6,170.6,170.3,170.0,169.8$, $167.8,140.2$, 138.8, 138.4, 128.4, 128.3, 128.0, 127.6, 127.6, 127.5, 126.9, 126.0, 104.9, 99.0, 82.1, 79.7, 75.3, 75.0, 74.0, 73.2, 71.7, 71.6, 70.3, 68.8, 67.1, 62.0, 61.6, 57.0, 53.0, 48.2, 37.3, 23.0, 21.4, 20.8, 20.8; LRMS (FAB): 961 (M+Na $\left.{ }^{+}, 100\right)$; HRMS(FAB) Calcd for $\mathrm{C}_{48} \mathrm{H}_{59} \mathrm{NO}_{18} \mathrm{Na}\left(\mathrm{M}+\mathrm{Na}^{+}\right)$960.3630, found 960.3636 .

\section{Synthesis of [Methyl 5-acetamido-3,5-dideoxy- $\beta$-D-glycero- $\alpha$-D-galacto-2-nonulo} -pyranosonate]-(2 $\rightarrow$ 6)-methyl 2,3,4-tri- $O$-benzyl- $\beta$-D-galactopyranoside (9a).

A solution of $\mathbf{8 a}(166 \mathrm{mg}, 0.18 \mathrm{mmol})$ in dry methanol $(2 \mathrm{~mL})$ containing a catalytic amount of sodium methoxide was stirred at room temperature for $7 \mathrm{~h}$. After treatment with DOWEX-50 $\mathrm{H}^{+}$resin, the mixture was concentrated to give 9a $(136 \mathrm{mg}$ ) as an amorphous solid quantitatively.

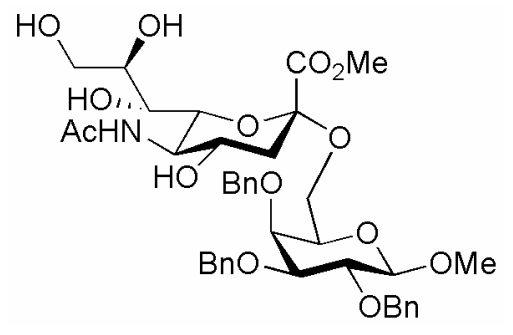

[Methyl 5-acetamido-3,5-dideoxy- $\beta$-D-glycero- $\alpha$-D-galacto-2-nonulo -pyranosonate]-(2 $\rightarrow$ 6)-methyl 2,3,4-tri- $O$-benzyl- $\beta$-D-galactopyranoside (9a). $[\alpha]_{\mathrm{D}}+9.8^{\circ}$ ( $c$ 0.2, MeOH); ${ }^{1} \mathrm{H}$ NMR (600 MHz, CD $\left.{ }_{3} \mathrm{OD}\right): \delta$ 7.37-7.22 (15H, m, ArH), $4.87\left(1 \mathrm{H}, \mathrm{d}, J=11.4 \mathrm{~Hz}, \mathrm{PhCH}_{2}\right), 4.85-4.78\left(1 \mathrm{H}, \mathrm{m}, \mathrm{PhCH}_{2}\right), 4.75-4.69\left(3 \mathrm{H}, \mathrm{m}, \mathrm{PhCH}_{2}\right)$, $4.60\left(1 \mathrm{H}, \mathrm{d}, J=11.4 \mathrm{~Hz}, \mathrm{PhCH}_{2}\right), 4.30(1 \mathrm{H}, \mathrm{d}, J=7.2 \mathrm{~Hz}, \mathrm{Gal}-\mathrm{H}-1), 3.99(1 \mathrm{H}, \mathrm{d}, J=1.8$ Hz, Gal-H-4), 3.94 (1H, dd, $J=5.8,9.3$ Hz, Gal-H-6a), 3.85-3.80 (2H, m, NeuAc-H-8 and NeuAc H-9a), $3.78(1 \mathrm{H}, \mathrm{t}, J=10.2 \mathrm{~Hz}, \mathrm{NeuAc}-\mathrm{H}-5), 3.71\left(3 \mathrm{H}, \mathrm{s}, \mathrm{COOCH}_{3}\right)$, 3.70-3.61 (5H, m, Gal-H-5, NeuAc-H-4, Gal-H-2, NeuAc-H-9b, Gal-H-3), 3.59 (1H, dd, $J=1.5,10.5 \mathrm{~Hz}, \mathrm{NeuAc-H}-6), 3.55-3.48$ (5H, m, Gal-H-6b, OMe, NeuAc-H-7), 2.68 (1H, 
$\mathrm{dd}, J=4.7,12.8 \mathrm{~Hz}$, NeuAc-H-3eq), 2.00 (3H,s, NAc), $1.73(1 \mathrm{H}, \mathrm{dd}, J=12.3,12.6 \mathrm{~Hz}$, NeuAc-H-3ax); ${ }^{13}$ C NMR (150 MHz, $\left.\mathrm{CD}_{3} \mathrm{OD}\right): \oint 175.3,170.8,140.3,140.3,140.1,129.5$, 129.4, 129.3, 129.3, 129.1, 129.0, 128.8, 128.7, 128.7, 106.4, 100.4, 83.4, 80.8, 76.1, 75.9, 75.1, 75.1, 74.4, 74.1, 72.5, 70.3, 68.7, 64.9, 63.6, 57.5, 53.9, 53.5, 41.8, 22.9; LRMS (FAB): $770\left(\mathrm{M}+\mathrm{H}^{+}, 100\right)$; HRMS(FAB) Calcd for $\mathrm{C}_{40} \mathrm{H}_{52} \mathrm{NO}_{14}\left(\mathrm{M}+\mathrm{H}^{+}\right) 770.3388$, found 770.3383

\section{Synthesis of [Methyl 5-acetamido-3,5-dideoxy- $\beta$-D-glycero- $\beta$-D-galacto-2-nonulo} -pyranosonate]-(2 $\rightarrow 6)$-methyl 2,3,4-tri- $O$-benzyl- $\beta$-D-galactopyranoside (9b).

A solution of $\mathbf{8 b}(306 \mathrm{mg}, 0.33 \mathrm{mmol})$ in dry methanol $(3 \mathrm{~mL})$ containing a catalytic amount of sodium methoxide was stirred at room temperature for $7 \mathrm{~h}$. After treatment with DOWEX-50 $\mathrm{H}^{+}$resin, the mixture was concentrated to give $9 \mathbf{b}(251 \mathrm{mg})$ as an amorphous solid quantitatively.

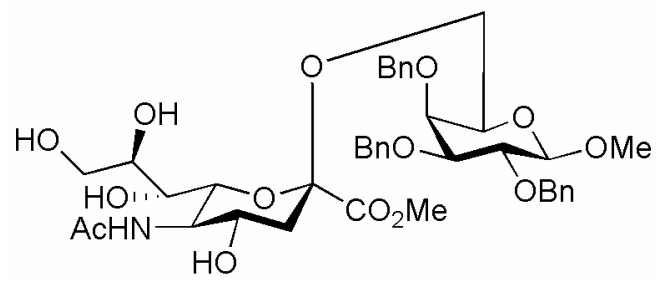

[Methyl 5-acetamido-3,5-dideoxy- $\beta$-D-glycero- $\beta$-D-galacto-2-nonulo -pyranosonate]-(2 $\rightarrow$ 6)-methyl 2,3,4-tri- $O$-benzyl- $\beta$-D-galactopyranoside $(9 \mathrm{~b})$. $[\alpha]_{\mathrm{D}}-7.3^{\circ}$ ( $c$ 0.1, MeOH); ${ }^{1} \mathrm{H}$ NMR (600 MHz, $\left.\mathrm{CD}_{3} \mathrm{OD}\right): \delta$ 7.36-7.23 (15H, m, ArH), 4.87-4.75 (2H, m, $\left.\mathrm{PhCH}_{2}\right), 4.74-4.68\left(3 \mathrm{H}, \mathrm{m}, \mathrm{PhCH}_{2}\right), 4.62\left(1 \mathrm{H}, \mathrm{d}, J=11.4 \mathrm{~Hz}, \mathrm{PhCH}_{2}\right)$, $4.27(1 \mathrm{H}, \mathrm{d}, J=7.8 \mathrm{~Hz}$, Gal-H-1), 4.03 (1H, m, NeuAc-H-4), 3.99 (1H, d, $J=2.9 \mathrm{~Hz}$, Gal-H-4), 3.84-3.78 (4H, m, NeuAc-H-5, NeuAc-H-9a, Gal-H-5 and Gal-H-6a), 3.77 (3H, s, $\left.\mathrm{COOCH}_{3}\right), 3.73(1 \mathrm{H}, \mathrm{m}, \mathrm{NeuAc}-\mathrm{H}-8), 3.69(1 \mathrm{H}, \mathrm{dd}, J=4.9,11.1 \mathrm{~Hz}, \mathrm{Gal}-\mathrm{H}-6 \mathrm{~b}), 3.65$ (1H, dd, $J=7.7,9.7$ Hz, Gal-H-2), 3.63-3.58 (2H, m, NeuAc-H-6 and NeuAc-H-9b), 3.56 $(1 \mathrm{H}, \mathrm{dd}, J=2.9,9.8 \mathrm{~Hz}, \mathrm{Gal}-\mathrm{H}-3), 3.54(3 \mathrm{H}, \mathrm{s}, \mathrm{OMe}), 3.49(1 \mathrm{H}, \mathrm{d}, J=9.1 \mathrm{~Hz}$, NeuAc-H-7), 2.40 (1H, dd, $J=4.9,13.0$ Hz, NeuAc-H-3eq), 2.00 (3H, s, NAc), 1.67 (1H, $\mathrm{dd}, J=11.3,13.0 \mathrm{~Hz}, \mathrm{NeuAc}-\mathrm{H}-3 \mathrm{ax}) ;{ }^{13} \mathrm{C} \mathrm{NMR}\left(125 \mathrm{MHz}, \mathrm{CD}_{3} \mathrm{OD}\right): \delta 175.0,170.7$, 140.3, 140.2, 140.0, 129.5, 129.4, 129.4, 129.3, 129.0, 128.8, 128.7, 106.3, 100.4, 83.4, 80.7, 76.3, 76.2, 76.1, 75.5, 74.0, 72.7, 71.5, 70.2, 67.8, 65.1, 65.0, 57.5, 54.2, 53.4, 41.6, 22.9; LRMS (FAB): $770\left(\mathrm{M}+\mathrm{H}^{+}, 100\right)$; $\mathrm{HRMS}(\mathrm{FAB})$ Calcd for $\mathrm{C}_{40} \mathrm{H}_{52} \mathrm{NO}_{14}\left(\mathrm{M}+\mathrm{H}^{+}\right)$ 770.3388 , found 770.3386

Synthesis of [Methyl 5-acetamido-3,5-dideoxy- $\beta$-D-glycero- $\alpha$-D-galacto-2-nonulo -pyranosonate]-(2 $\rightarrow$ 6)-methyl $\beta$-D-galactopyranoside (10a). 
A solution of 9a $(136 \mathrm{mg}, 0.18 \mathrm{mmol})$ in dry methanol $(25 \mathrm{~mL})$ was hydrogenolysed over $\mathrm{Pd} / \mathrm{C}(10 \%, 85 \mathrm{mg})$ under $4 \mathrm{~atm}$ until TLC showed that the reaction was complete (18 h). The mixture was filtered, concentrated, subjected to reverse phase HPLC using $\mathrm{H}_{2} \mathrm{O}$-acetonitrile gradient, then lyophilised to give $56 \mathrm{mg}(\mathrm{y} .63 \%)$ of 10a as an amorphous solid.

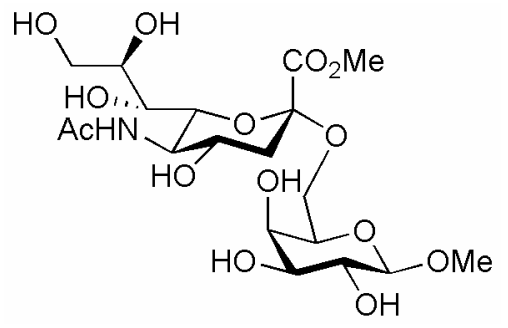

[Methyl 5-acetamido-3,5-dideoxy- $\beta$-D-glycero- $\alpha$-D-galacto-2-nonulo -pyranosonate]-(2 $\rightarrow 6)$-methyl $\boldsymbol{\beta}$-D-galactopyranoside (10a).

$[\alpha]_{\mathrm{D}}-36.6^{\circ}$ (c 1.0, MeOH); ${ }^{1} \mathrm{H}$ NMR $\left(600 \mathrm{MHz}, \mathrm{D}_{2} \mathrm{O}\right): \delta 4.36(1 \mathrm{H}, \mathrm{d}, J=7.8 \mathrm{~Hz}$, Gal-H-1), 4.06 (1H, dd, $J=7.2,10.2$ Hz, Gal-H-6a), 3.97 (1H, d, $J=3.6$ Hz, Gal-H-4), 3.94 (3H, s, $\left.\mathrm{COOCH}_{3}\right)$, 3.94-3.89 (4H, m, NeuAc-H-5, NeuAc-H-9a, NeuAc-H-6 and NeuAc-H-7), 3.86-3.80 (2H, m, Gal-H-5 and NeuAc-H-4), 3.77-3.67 (3H, m, Gal-H-6b, NeuAc-H-9b and Gal-H-3), 3.62 (3H, s, OMe), 3.62-3.60 (1H, m, NeuAc-H-8), 3.55 (1H, $\mathrm{dd}, J=7.8,9.9 \mathrm{~Hz}, \mathrm{Gal}-\mathrm{H}-2), 2.78(1 \mathrm{H}, \mathrm{dd}, J=4.2,12.6 \mathrm{~Hz}$, NeuAc-H-3eq), 2.09 (3H, s, NAc), $1.90(1 \mathrm{H}, \mathrm{dd}, J=12.3,12.6 \mathrm{~Hz}$, NeuAc-H-3ax $) ;{ }^{13} \mathrm{C} \mathrm{NMR}\left(150 \mathrm{MHz}, \mathrm{D}_{2} \mathrm{O}\right): \delta$ 175.4, 170.3, 104.3, 99.5, 73.6, 73.4, 73.1, 71.0, 69.0, 68.7, 67.6, 63.8, 63.6, 57.8, 53.9, 52.1, 39.4, 22.5; IR (DMSO- $d_{6}$ ) 1732, 1670, 1647, 1558, 1447, 1373, 1292, $1200 \mathrm{~cm}^{-1}$, LRMS (FAB): $500\left(\mathrm{M}+\mathrm{H}^{+}, 100\right)$; HRMS(FAB) Calcd for $\mathrm{C}_{19} \mathrm{H}_{34} \mathrm{NO}_{14}\left(\mathrm{M}+\mathrm{H}^{+}\right) 500.1979$, found 500.1974 .

\section{Synthesis of [Methyl 5-acetamido-3,5-dideoxy- $\beta$-D-glycero- $\beta$-D-galacto-2-nonulo -pyranosonate]-( $2 \rightarrow 6)$-methyl $\beta$-D-galactopyranoside (10b).}

A solution of $9 \mathbf{b}(251 \mathrm{mg}, 0.33 \mathrm{mmol})$ in dry methanol $(46 \mathrm{~mL})$ was hydrogenolysed over $\mathrm{Pd} / \mathrm{C}(10 \%, 155 \mathrm{mg})$ under $4 \mathrm{~atm}$ until TLC showed that the reaction was complete $(20 \mathrm{~h})$. The mixture was filtered, concentrated, subjected to reverse phase HPLC using $\mathrm{H}_{2} \mathrm{O}$-acetonitrile gradient, and then lyophilised to give $64 \mathrm{mg}$ (y. $39 \%$ ) of $\mathbf{1 0 b}$ as an amorphous solid. 


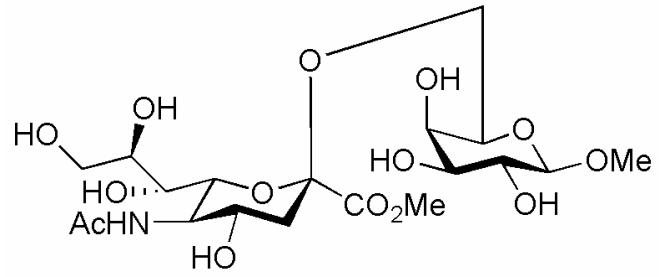

[Methyl 5-acetamido-3,5-dideoxy- $\beta$-D-glycero- $\beta$-D-galacto-2-nonulo -pyranosonate]-( $2 \rightarrow 6)$-methyl $\beta$-D-galactopyranoside $(10 \mathrm{~b})$.

$[\alpha]_{\mathrm{D}}-22.0^{\circ}(c 0.2, \mathrm{MeOH}) ;{ }^{1} \mathrm{H}$ NMR $\left(600 \mathrm{MHz}, \mathrm{D}_{2} \mathrm{O}\right): \delta 4.38(1 \mathrm{H}, \mathrm{d}, J=7.9 \mathrm{~Hz}$, Gal-H-1), 4.16 (1H, m, NeuAc-H-4), 4.00-3.95 (2H, m, NeuAc-H-5 and Gal-H-4), 3.93 $\left(3 \mathrm{H}, \mathrm{s}, \mathrm{COOCH}_{3}\right)$, 3.93-3.80 (5H, m, NeuAc-H-8, NeuAc-H-9a, NeuAc-H-7, Gal-H-6a and Gal-H-5), $3.73(1 \mathrm{H}, \mathrm{dd}, J=6.2,12.7 \mathrm{~Hz}, \mathrm{H}-9 \mathrm{~b}), 3.69(1 \mathrm{H}, \mathrm{dd}, J=3.4,9.9 \mathrm{~Hz}$, Gal-H-3), 3.67-3.61 (4H, m, NeuAc-H-6 and OMe), 3.61-3.51 (2H, m, Gal-H-6b and Gal-H-2), 2.53 (1H, dd, $J=4.8,13.1 \mathrm{~Hz}, \mathrm{NeuAc}-\mathrm{H}-3 \mathrm{eq}), 2.11$ (3H, s, NAc), 1.86 (1H, dd, $J=12.3,12.9 \mathrm{~Hz}$, NeuAc-H-3ax $) ;{ }^{13} \mathrm{C}$ NMR $\left(150 \mathrm{MHz}, \mathrm{D}_{2} \mathrm{O}\right): \delta 175.3,170.6,104.1,99.4$, 73.8, 73.1, 71.3, 71.0, 70.4, 69.4, 68.4, 66.7, 63.8, 63.4, 57.6, 54.0, 52.2, 39.5, 22.5; IR (DMSO- $d_{6}$ ) 1747, 1670, 1647, 1558, 1439, 1373, 1315, $1269 \mathrm{~cm}^{-1}$; LRMS (FAB): 500 $\left(\mathrm{M}+\mathrm{H}^{+}, 100\right)$; HRMS(FAB) Calcd for $\mathrm{C}_{19} \mathrm{H}_{34} \mathrm{NO}_{14}\left(\mathrm{M}+\mathrm{H}^{+}\right) 500.1979$, found 500.1990. 

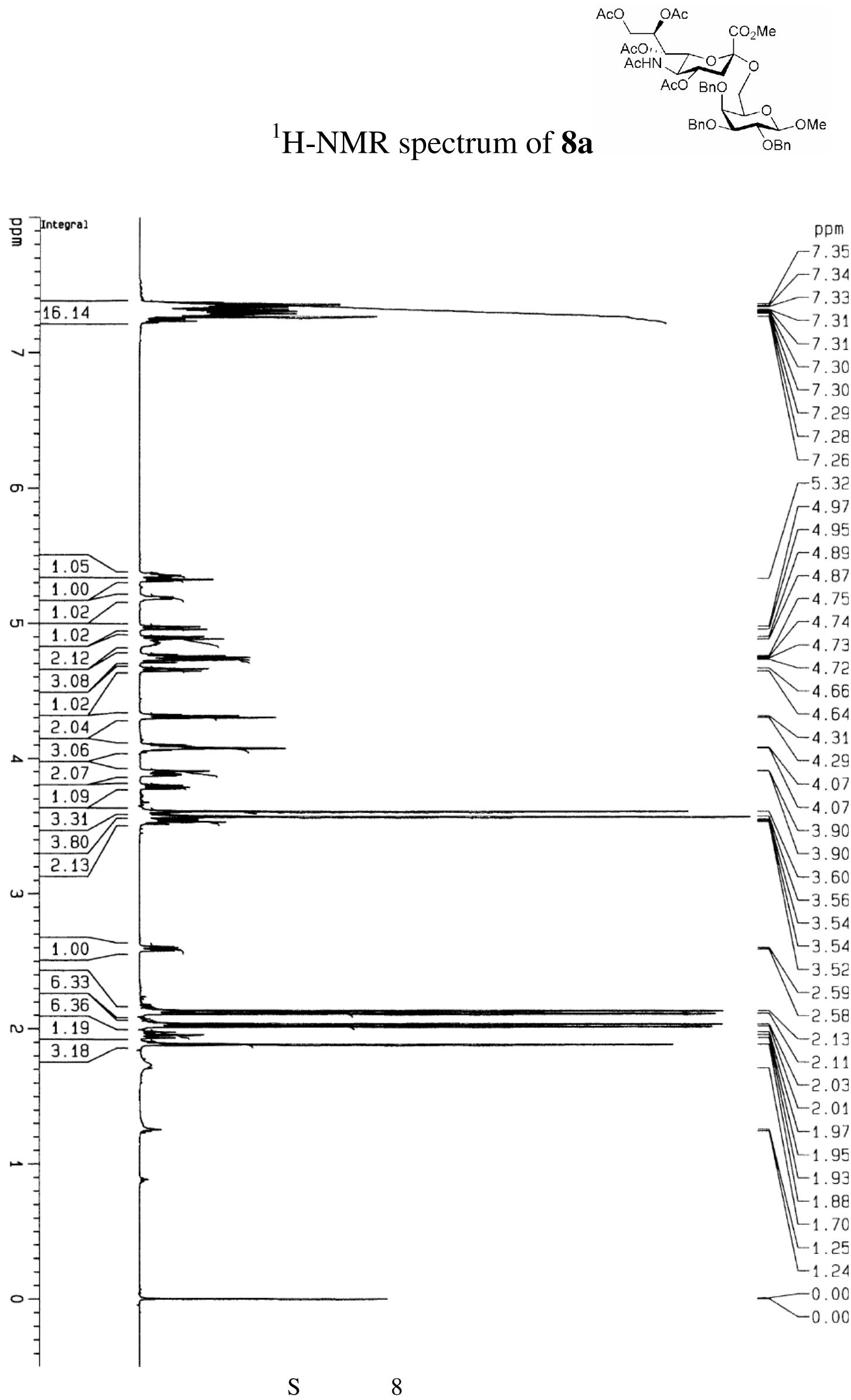


$$
\xi
$$


${ }^{1} \mathrm{H},{ }^{1} \mathrm{H}-\mathrm{COSY}$ spectrum of $\mathbf{8 a}$
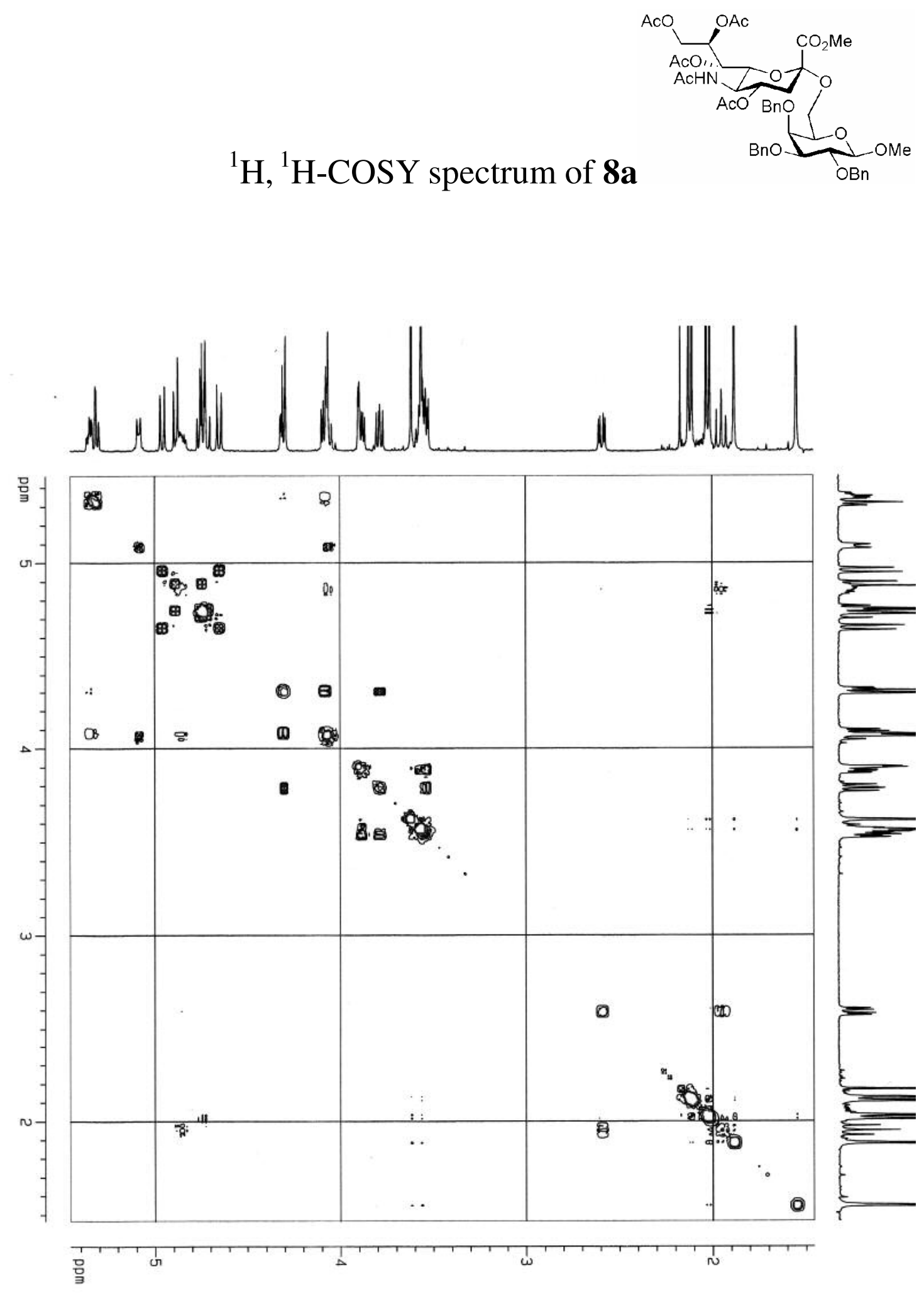

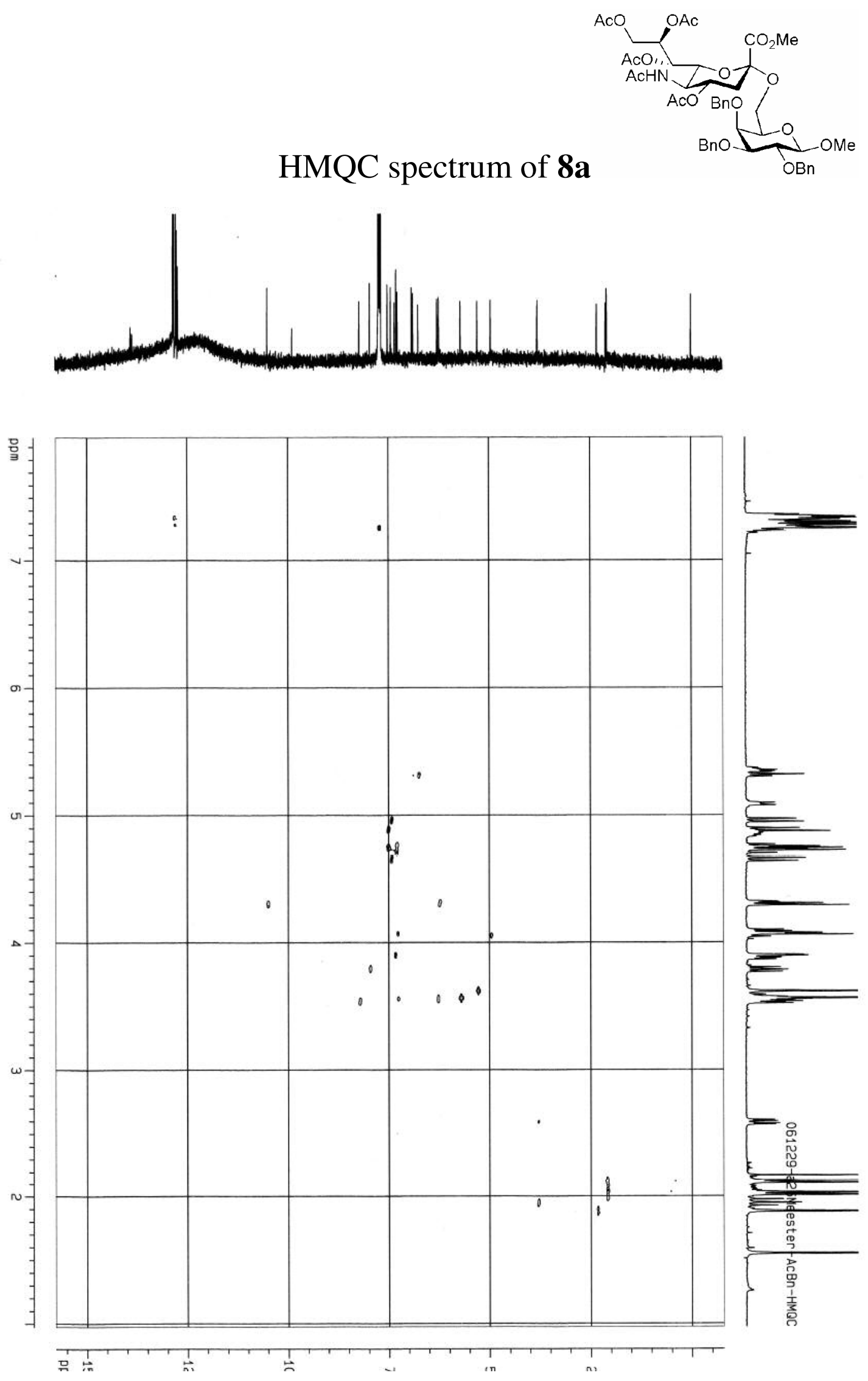

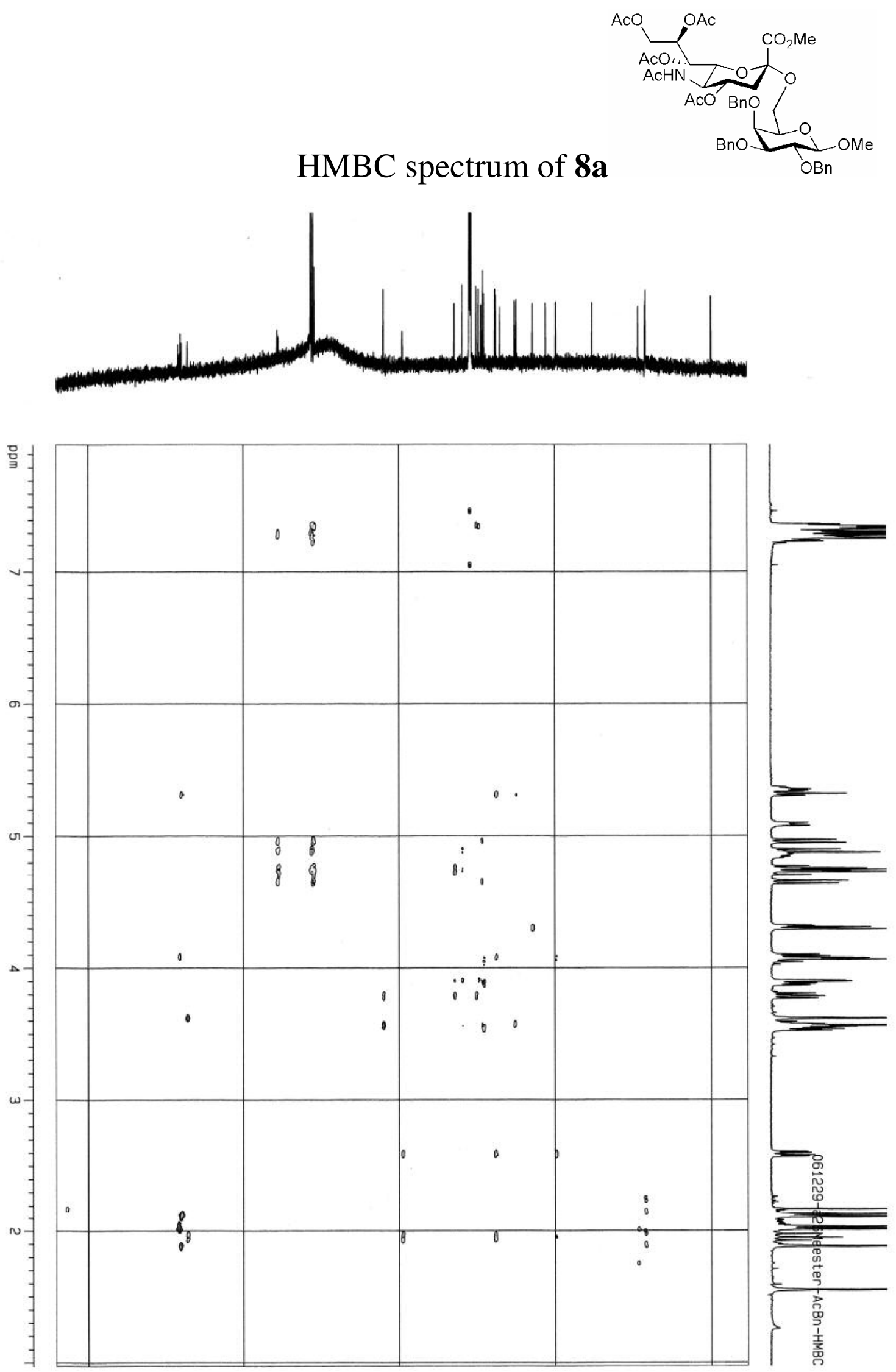

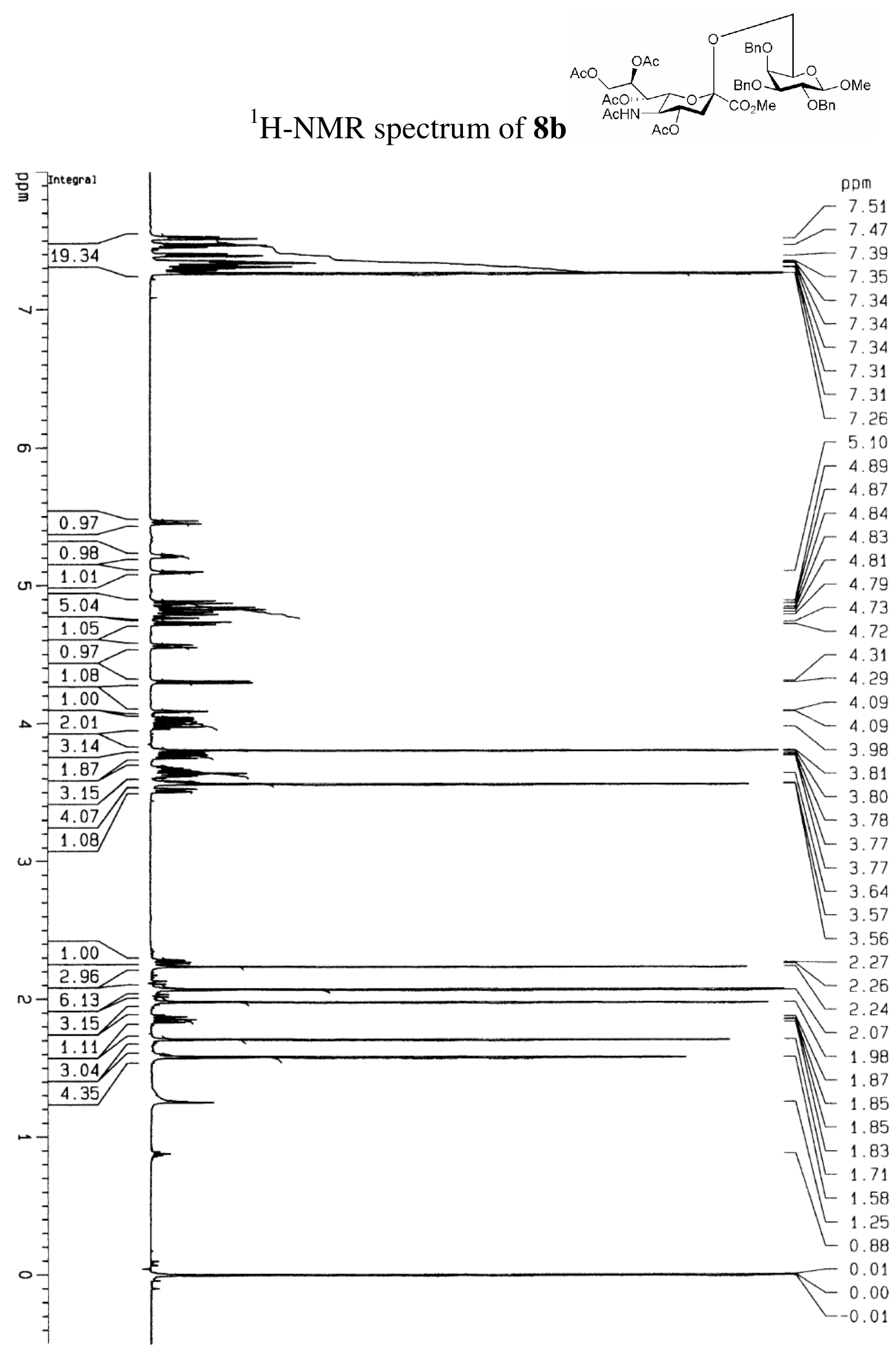


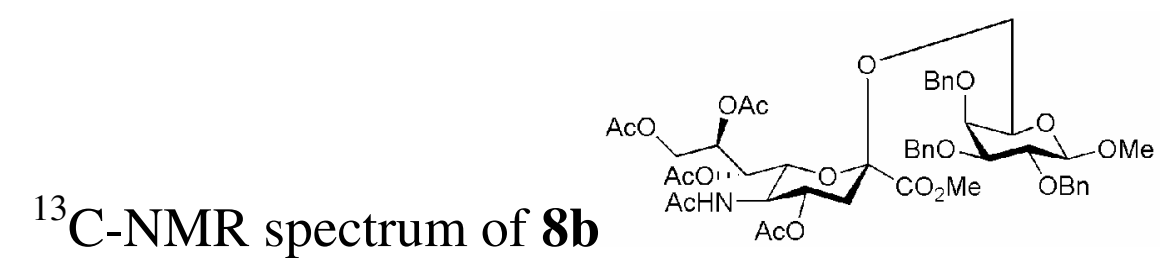

㰻

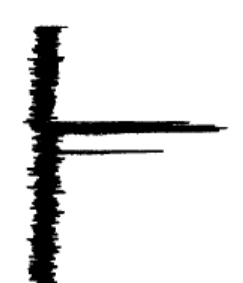

吕

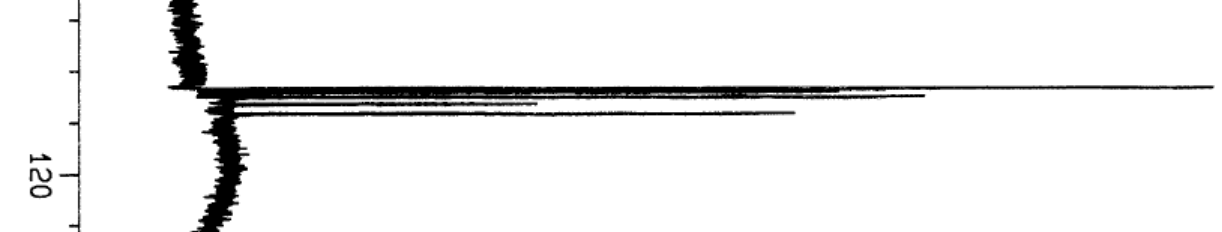

ก

。

ஜ

8

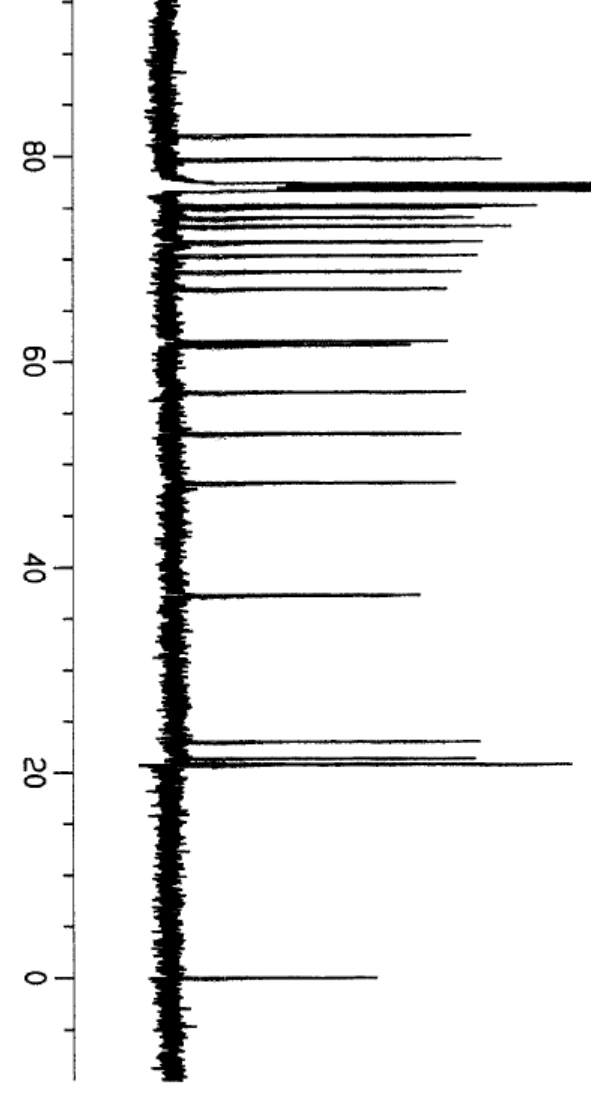




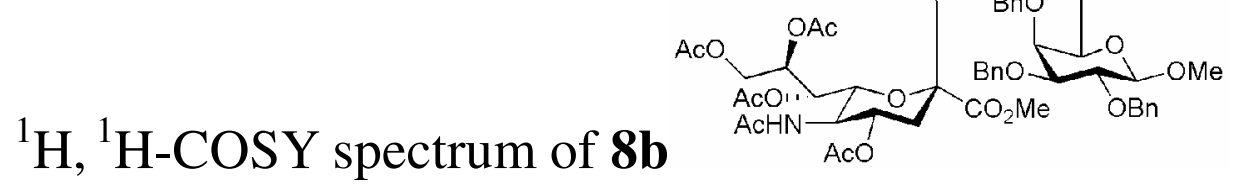

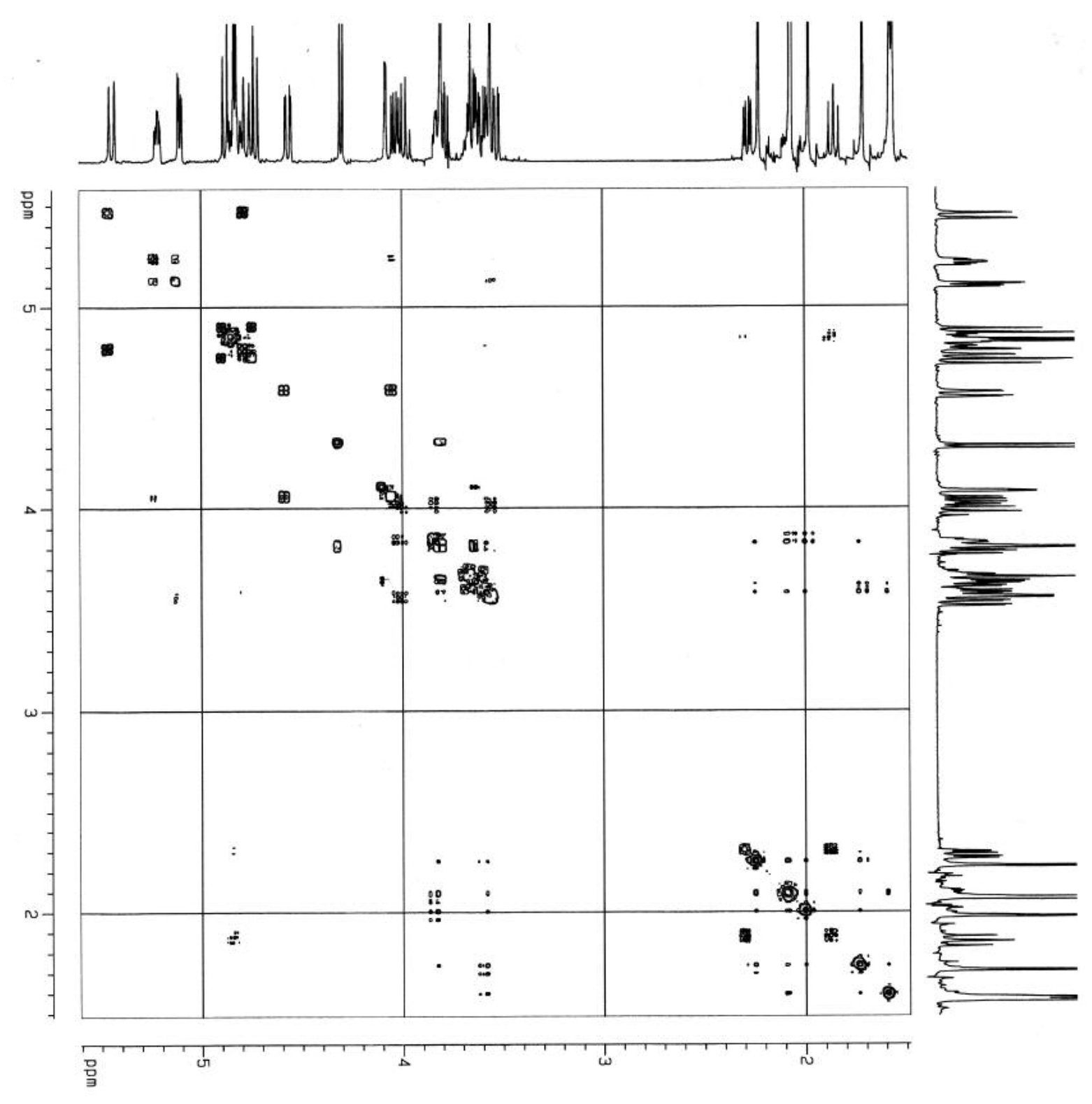




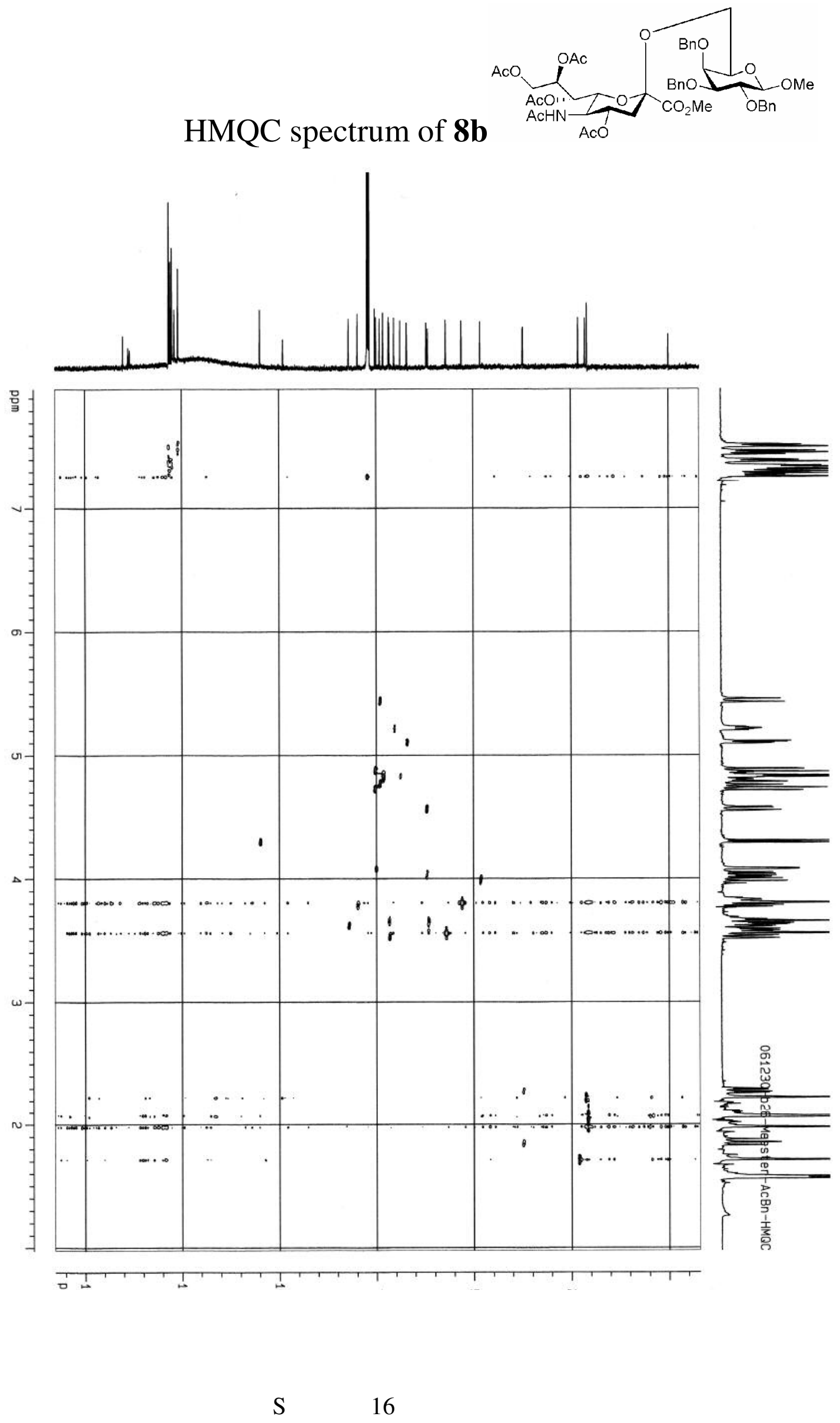



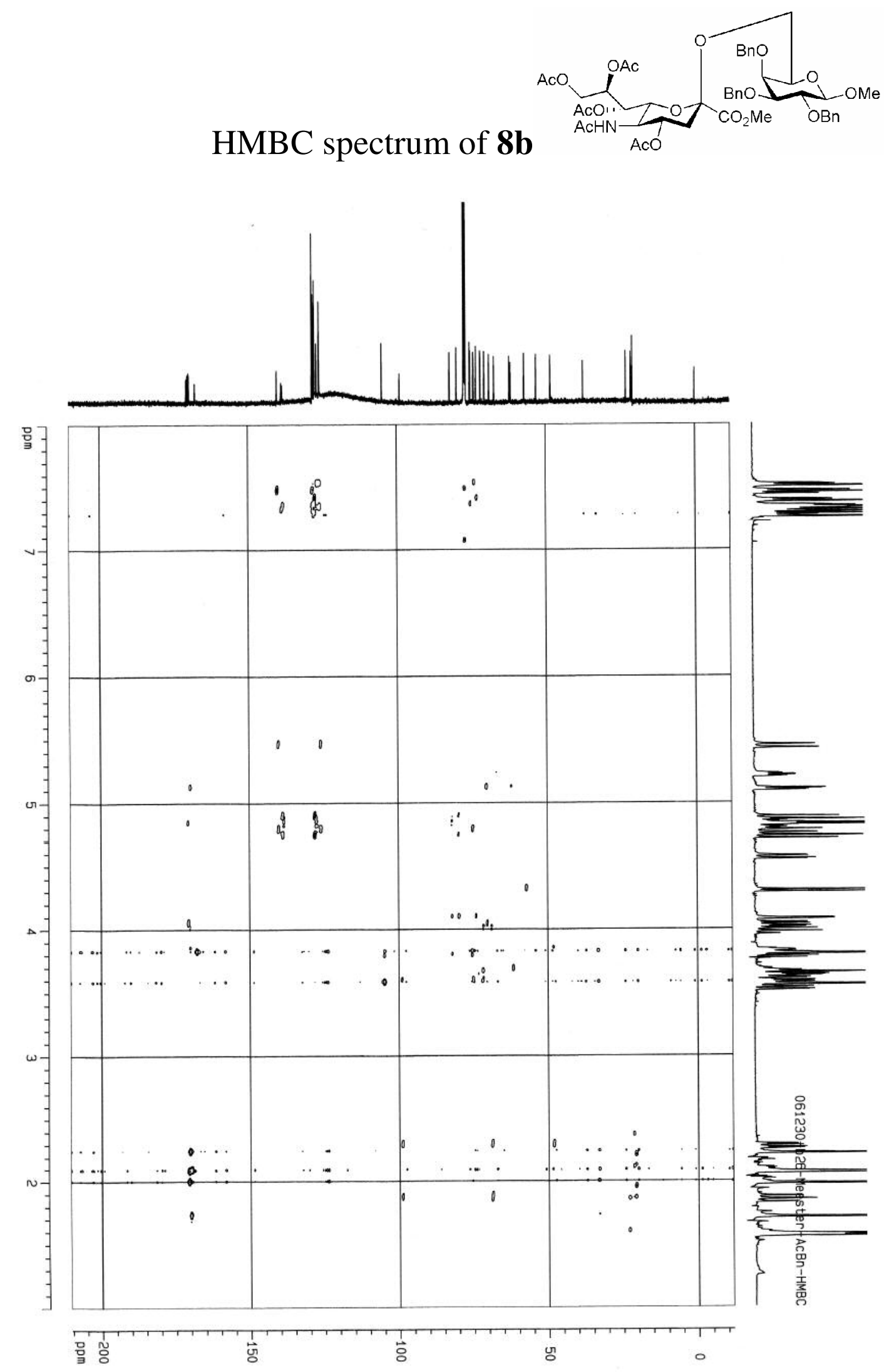

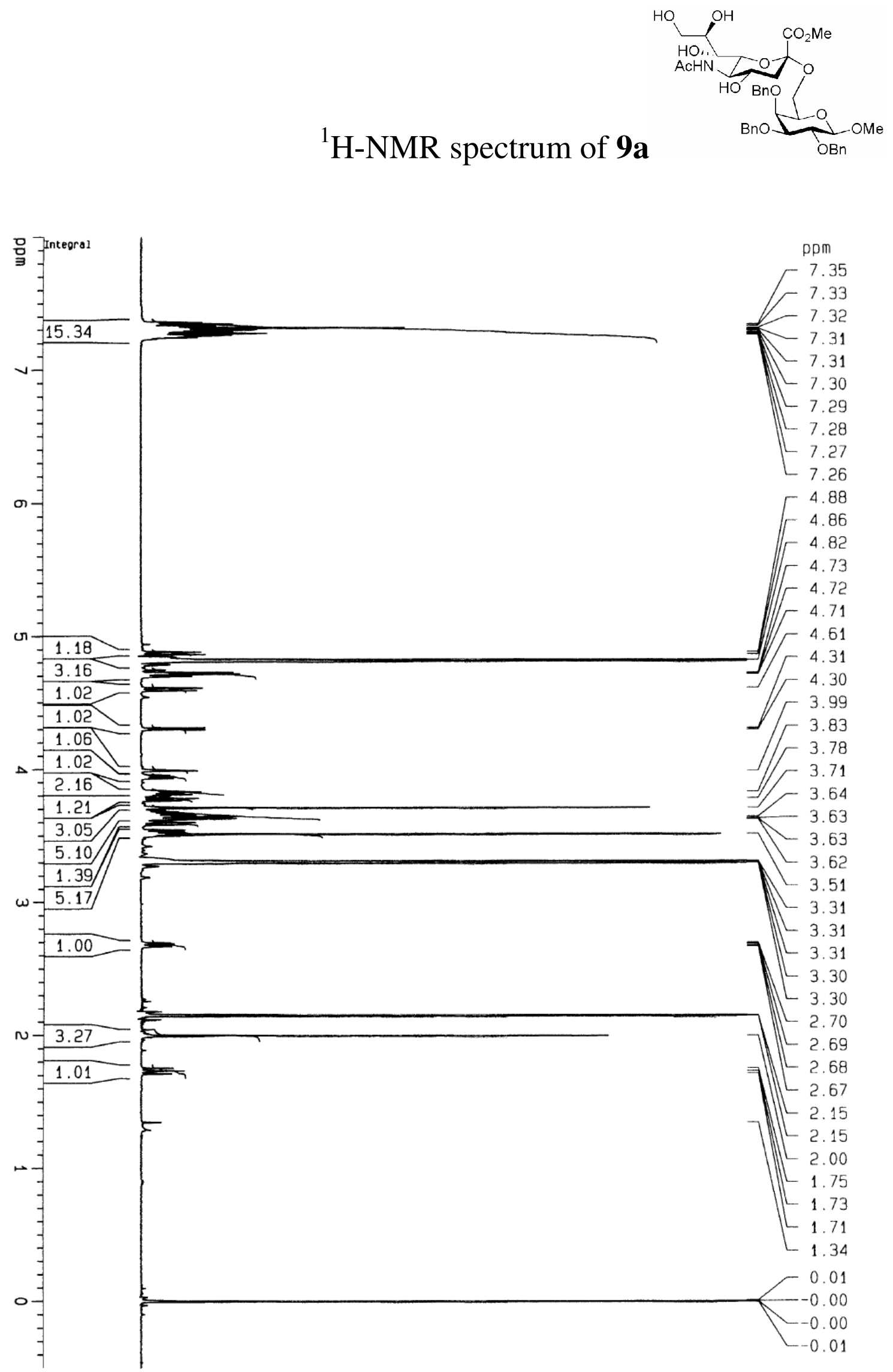

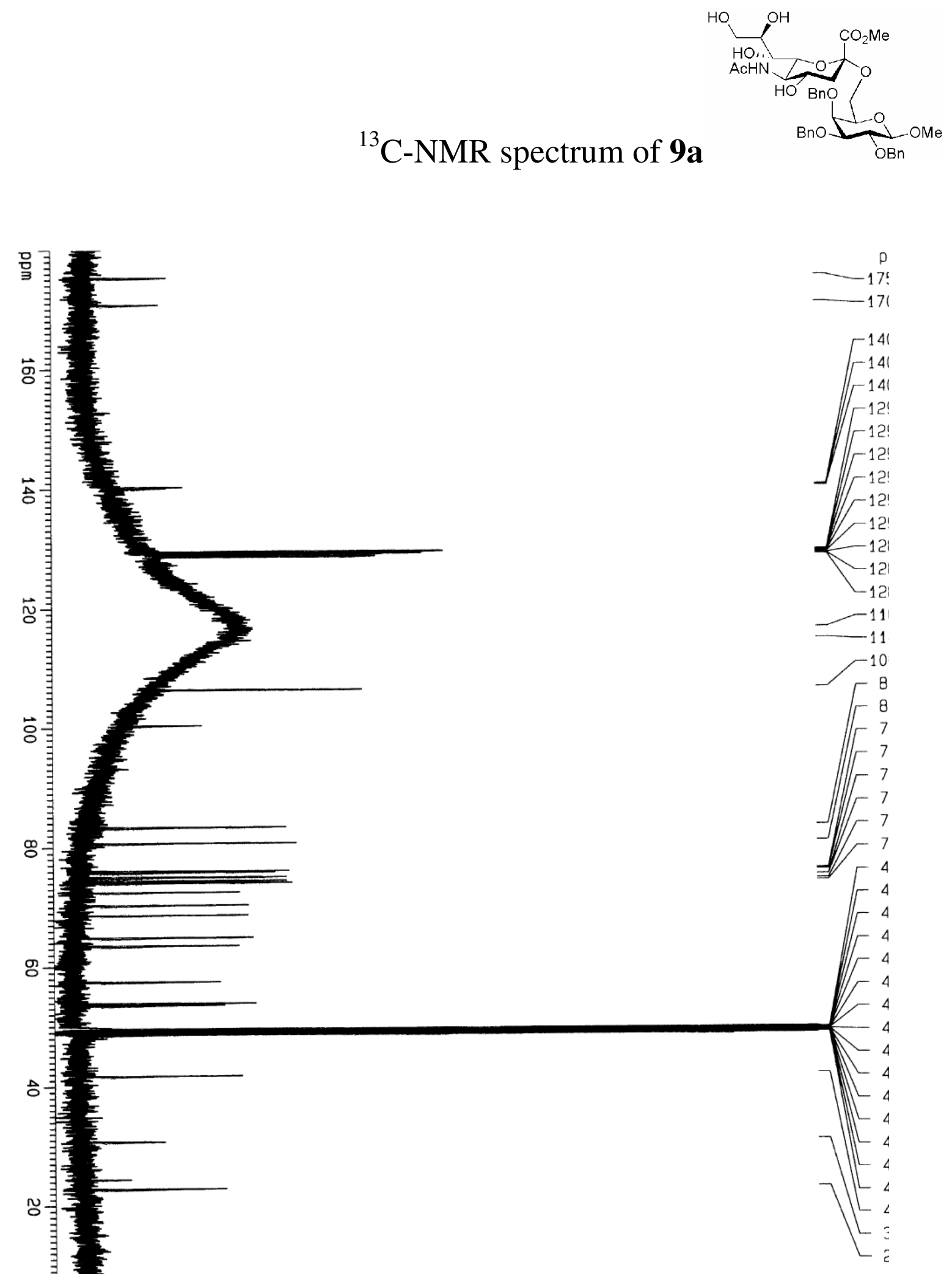

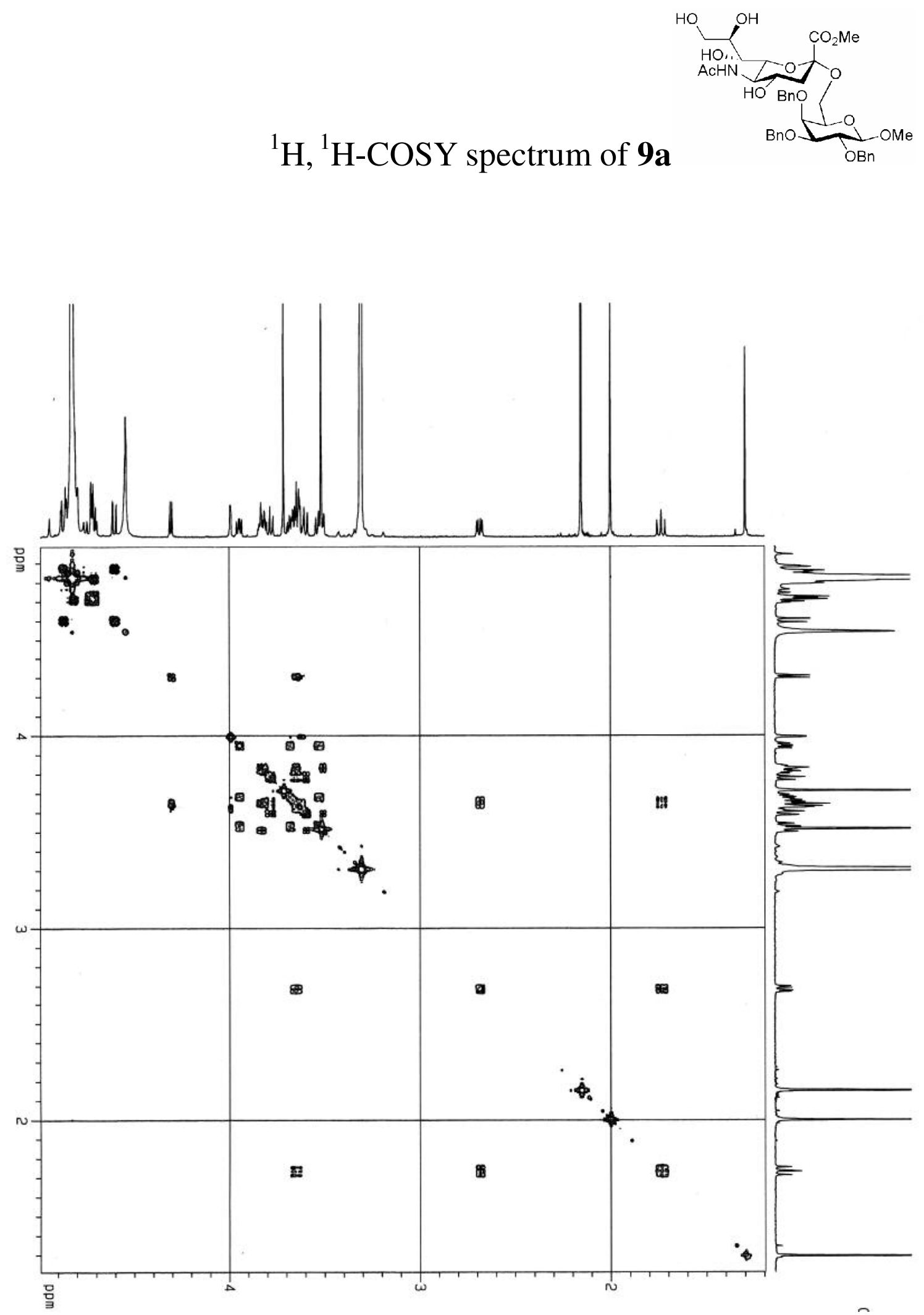

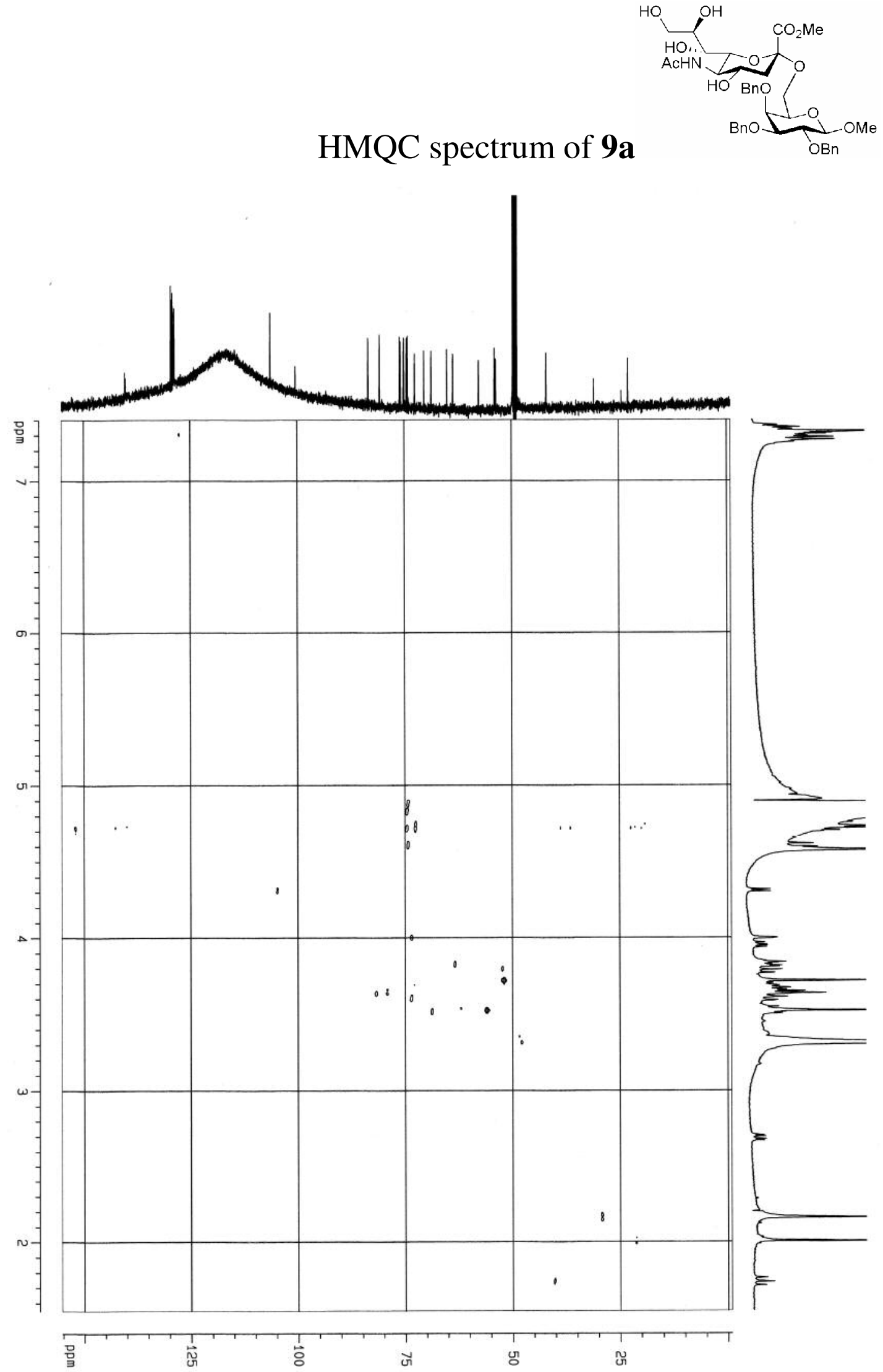

S

21 

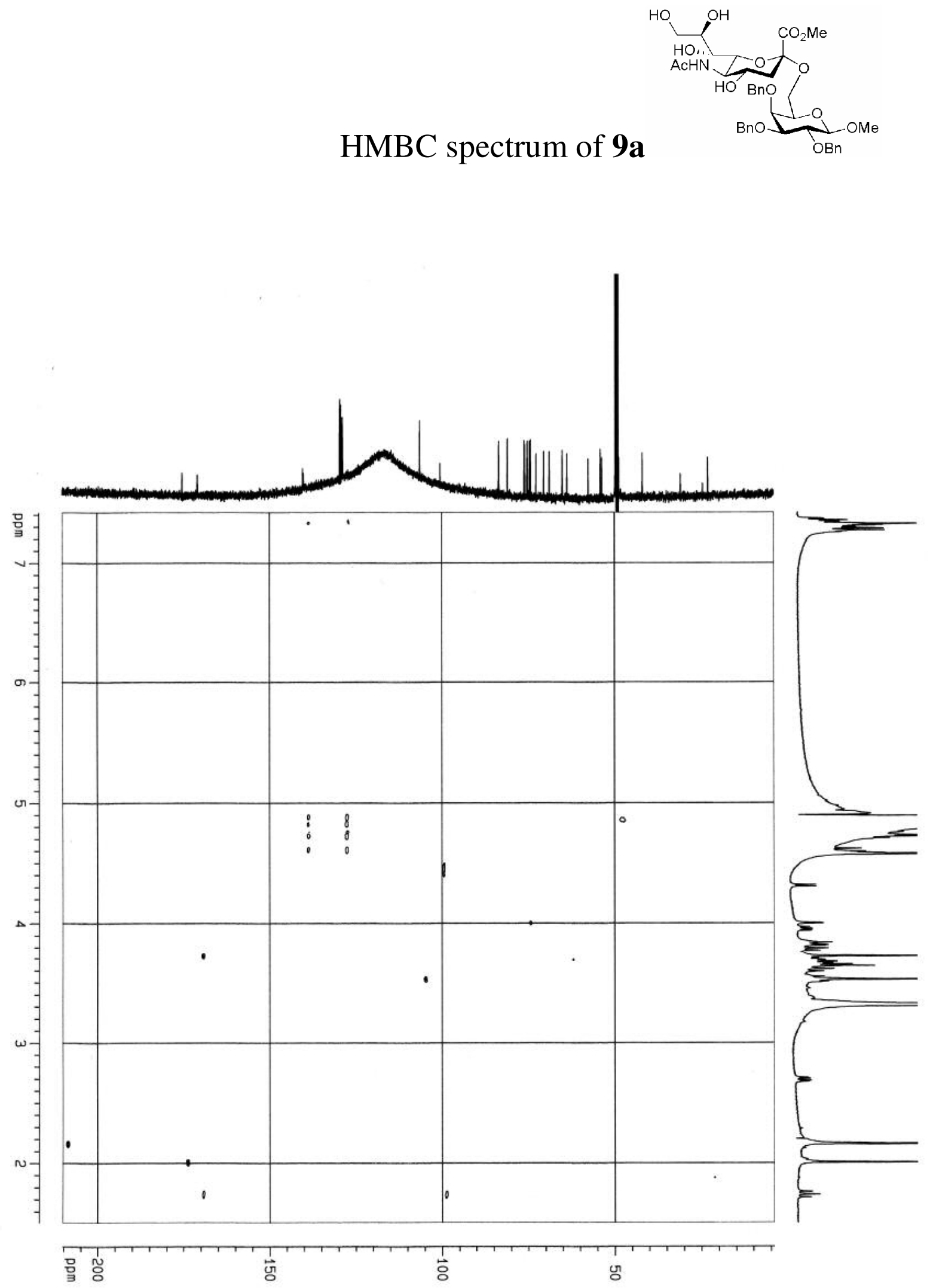

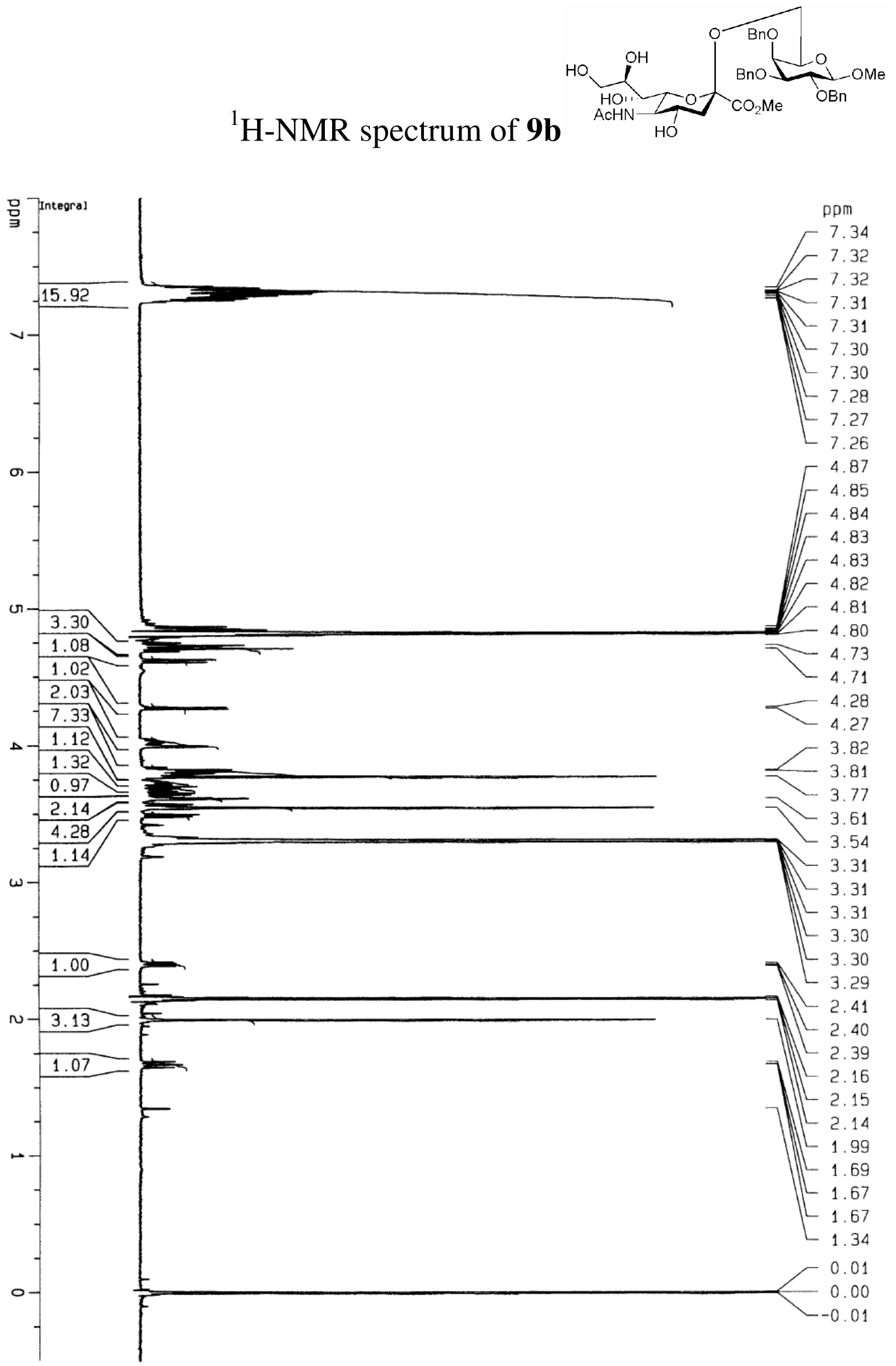

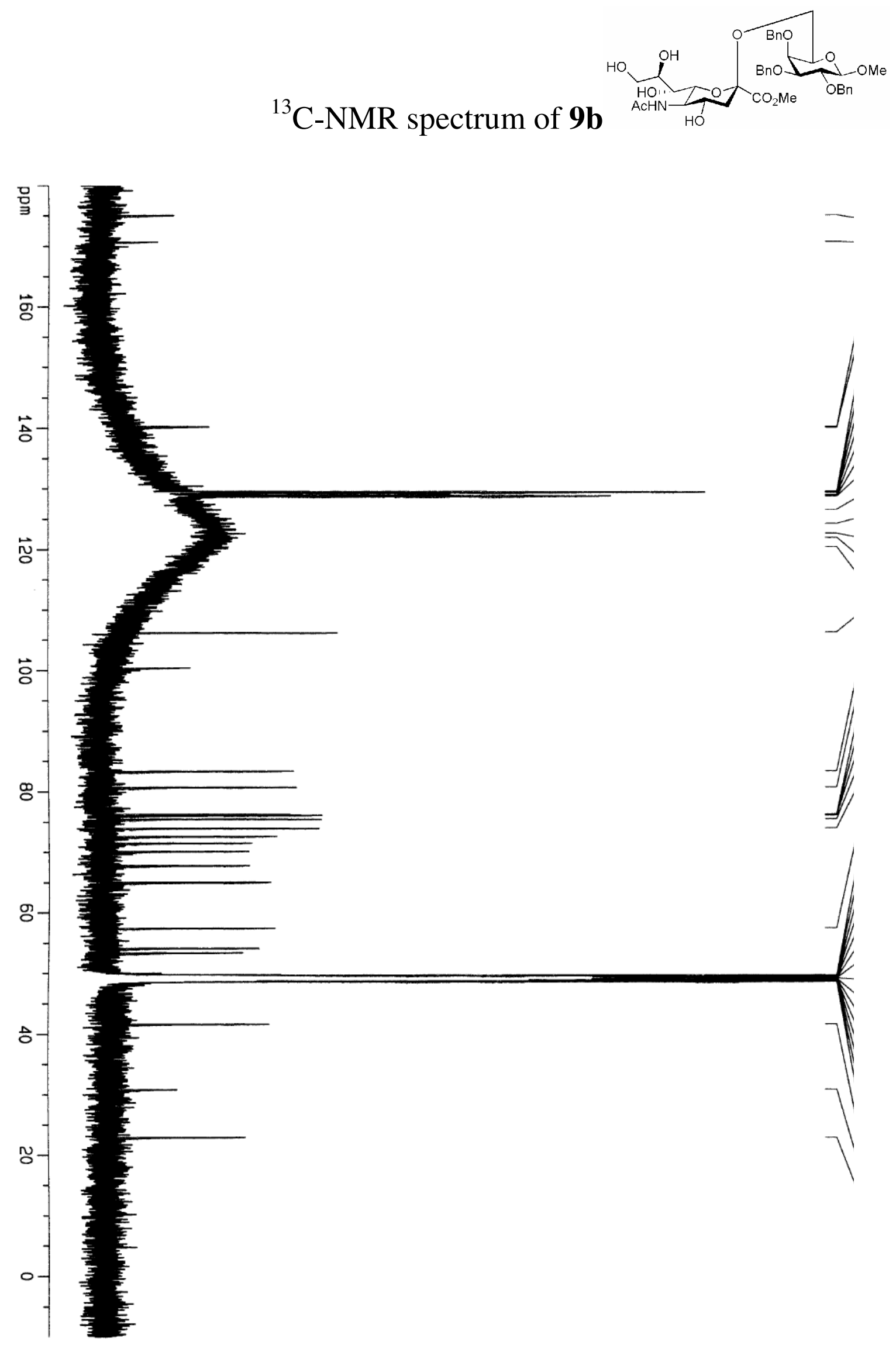
${ }^{1} \mathrm{H},{ }^{1} \mathrm{H}-\mathrm{COSY}$ spectrum of $\mathbf{9 b}$
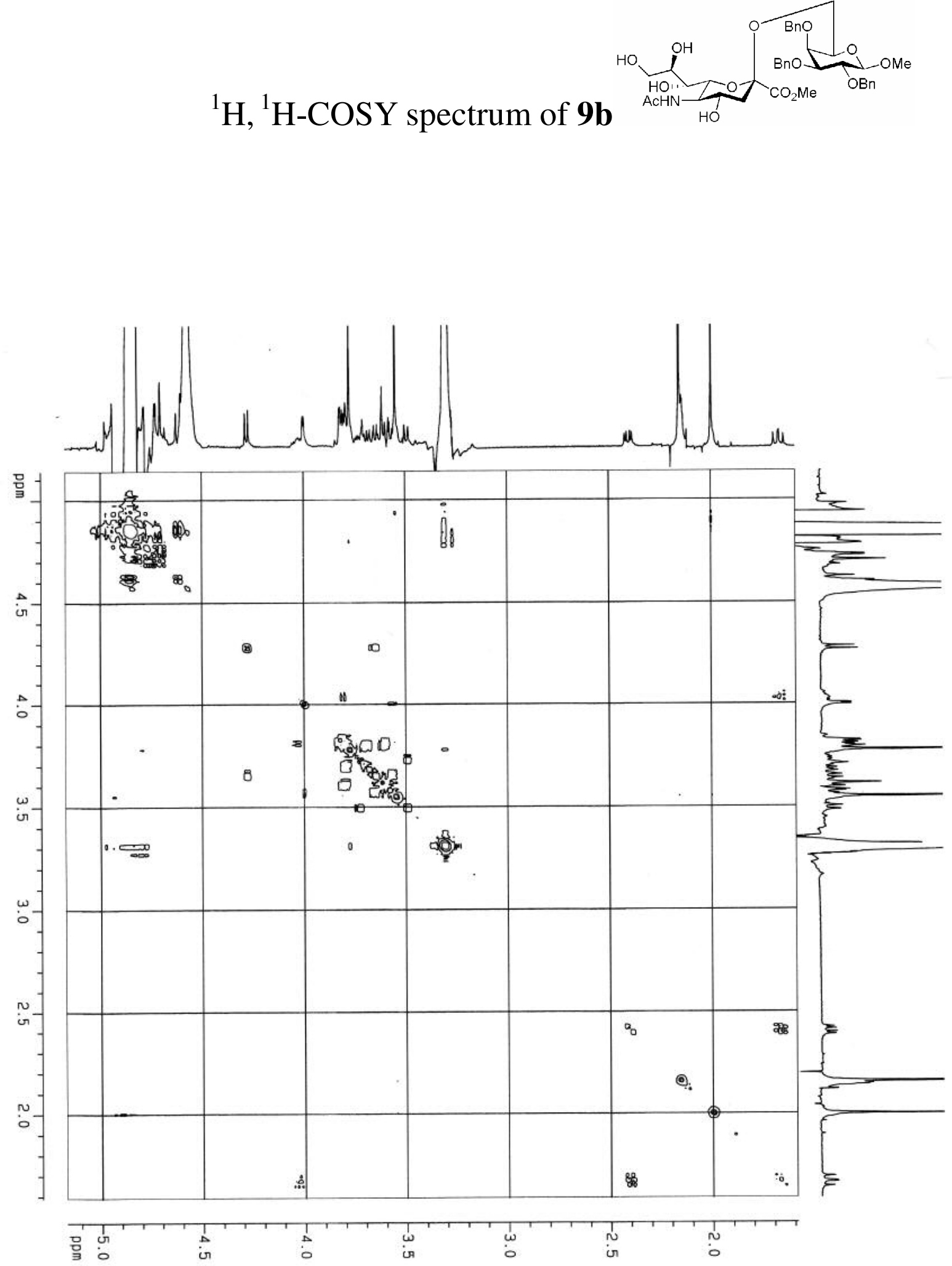

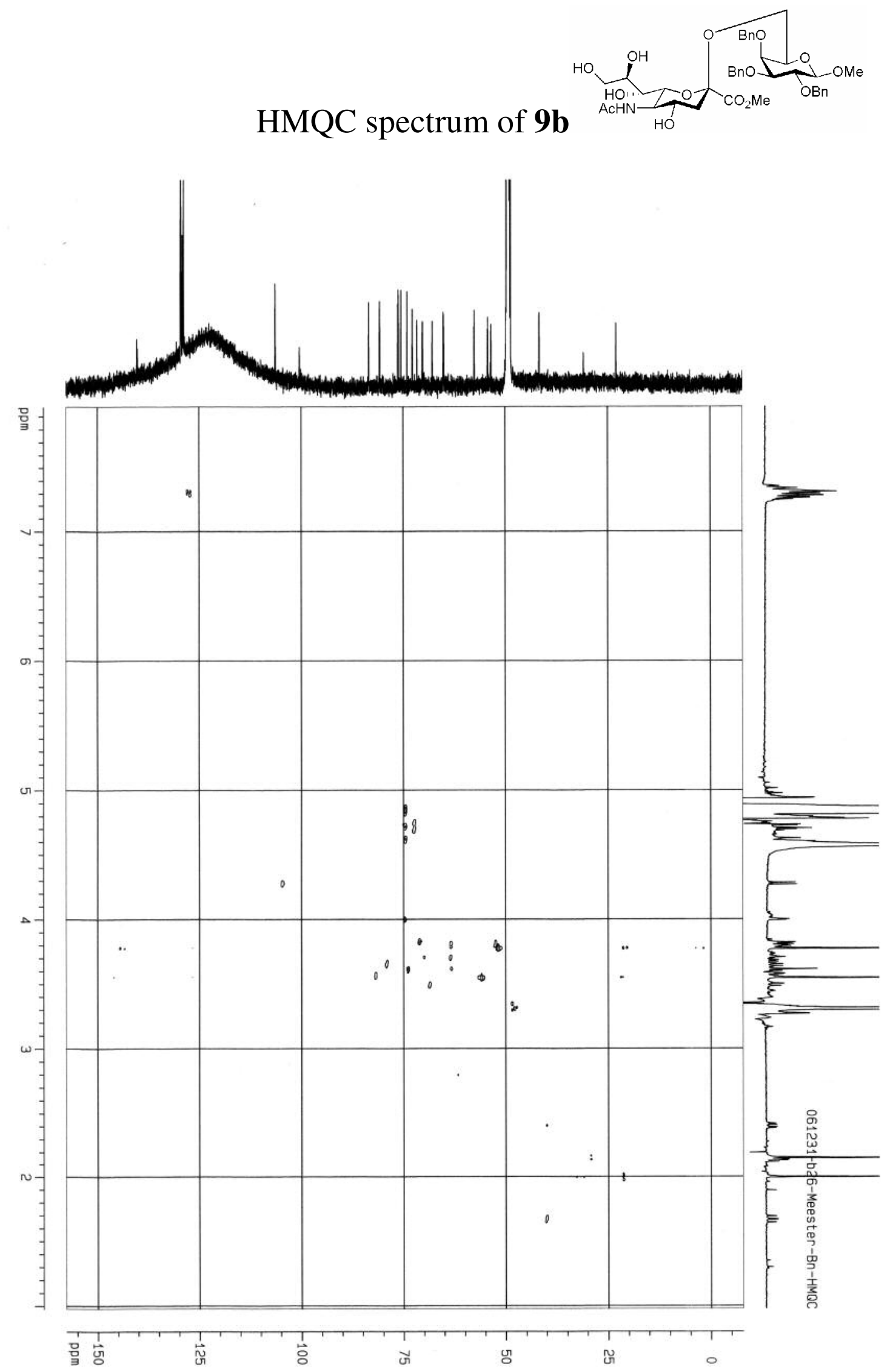


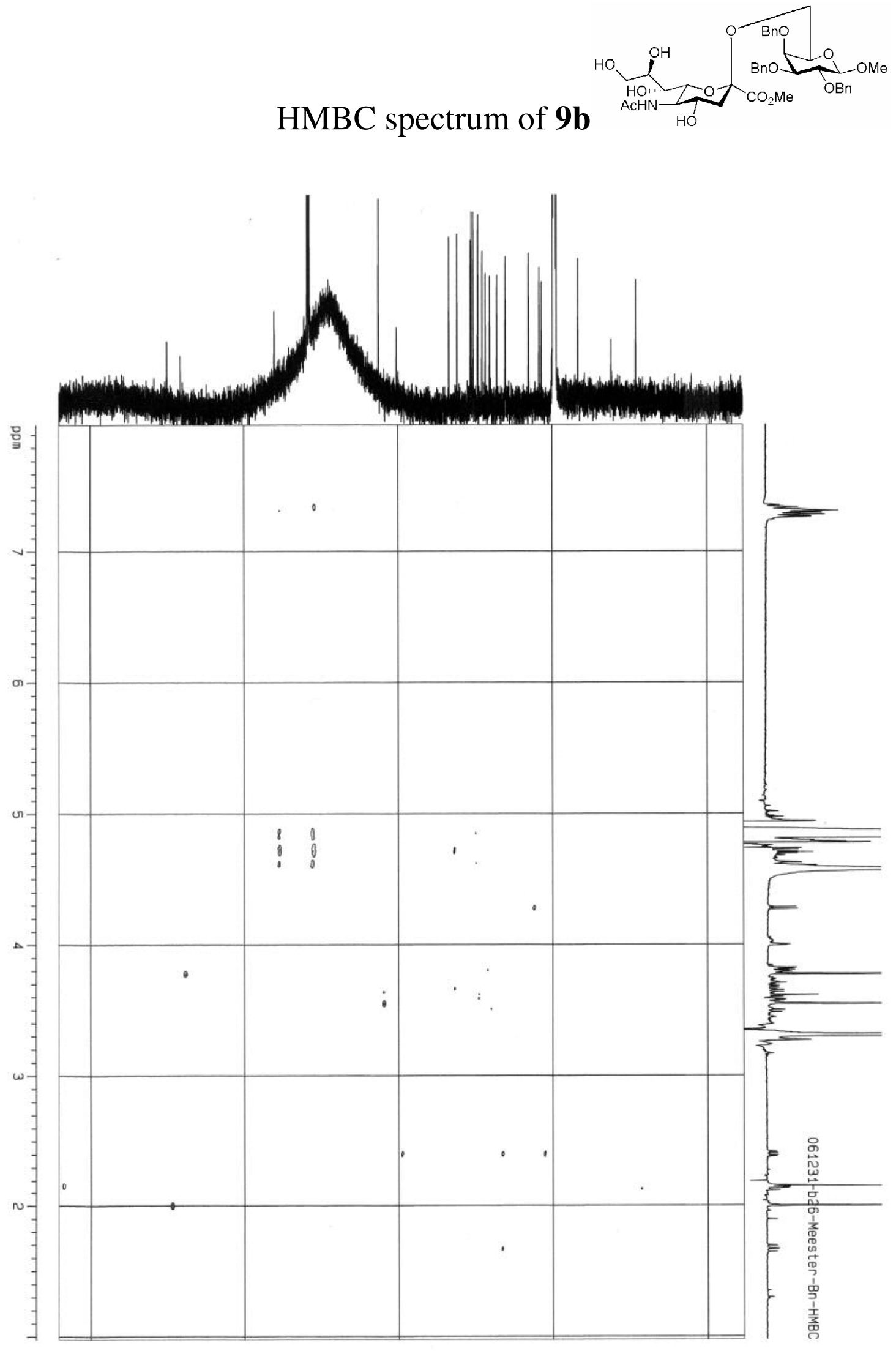




\section{${ }^{1} \mathrm{H}-\mathrm{NMR}$ spectrum of $\mathbf{1 0 a}$}
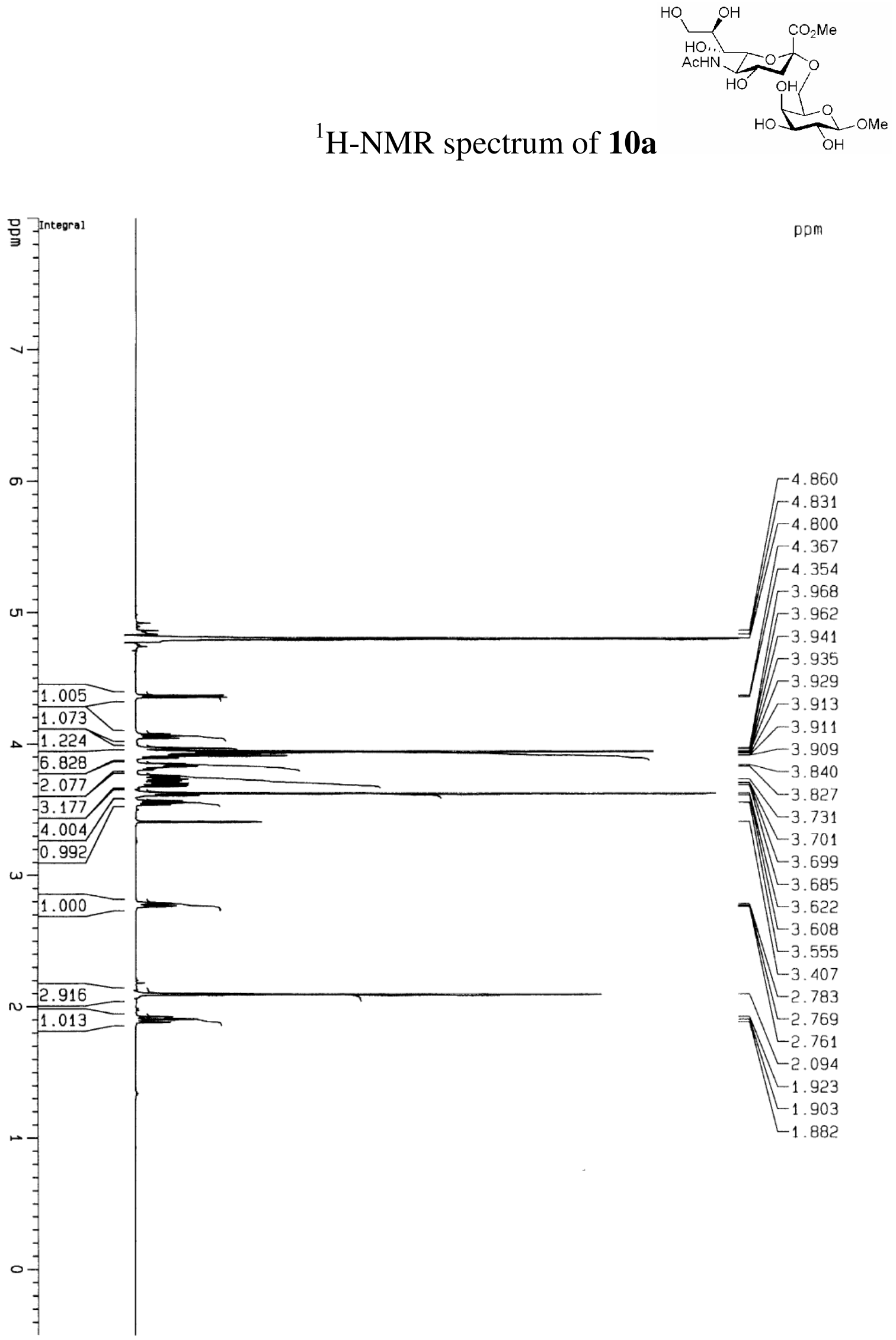

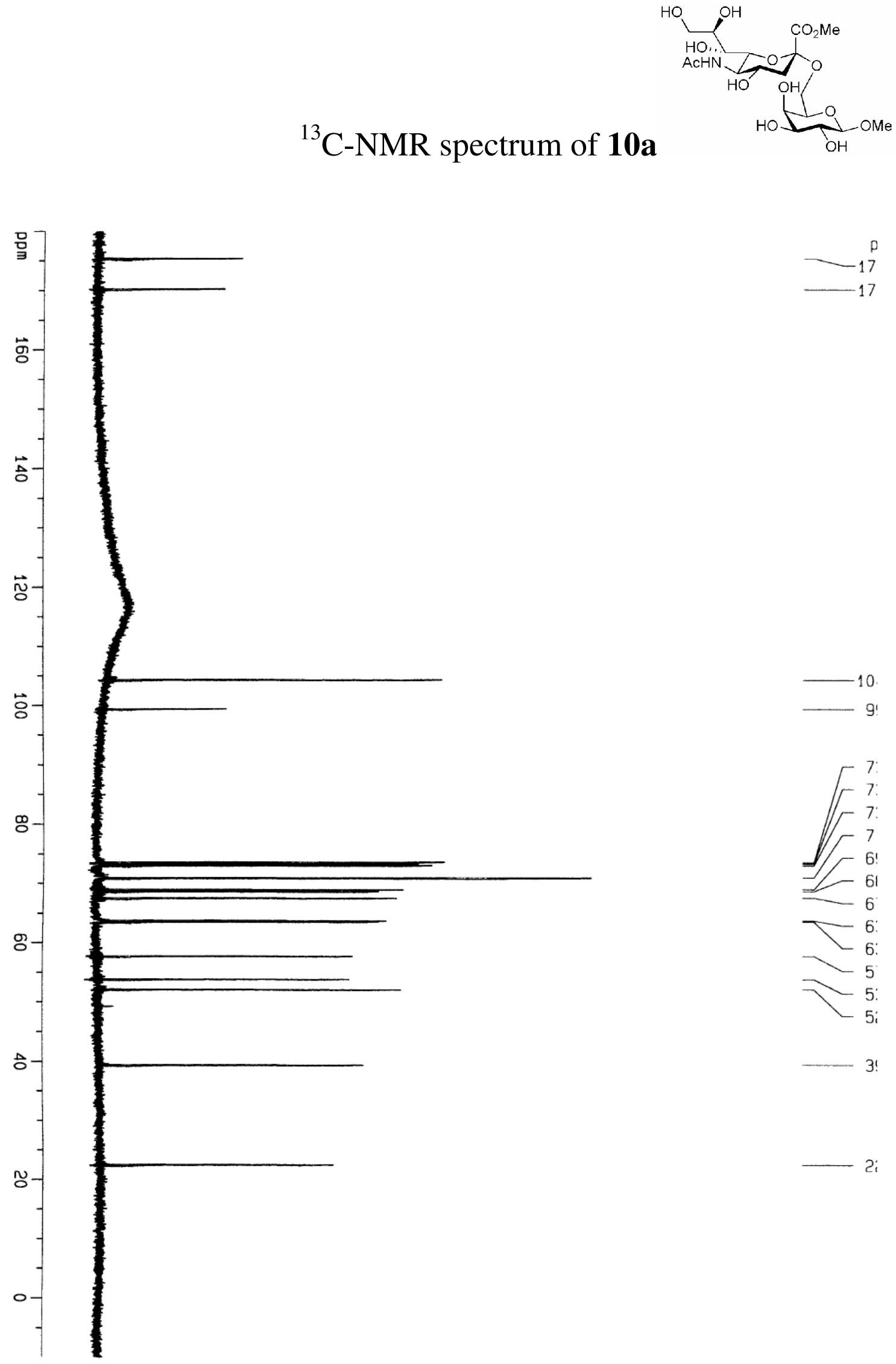

- 3 !

S

29 
${ }^{1} \mathrm{H},{ }^{1} \mathrm{H}-\mathrm{COSY}$ spectrum of $\mathbf{1 0 a}$
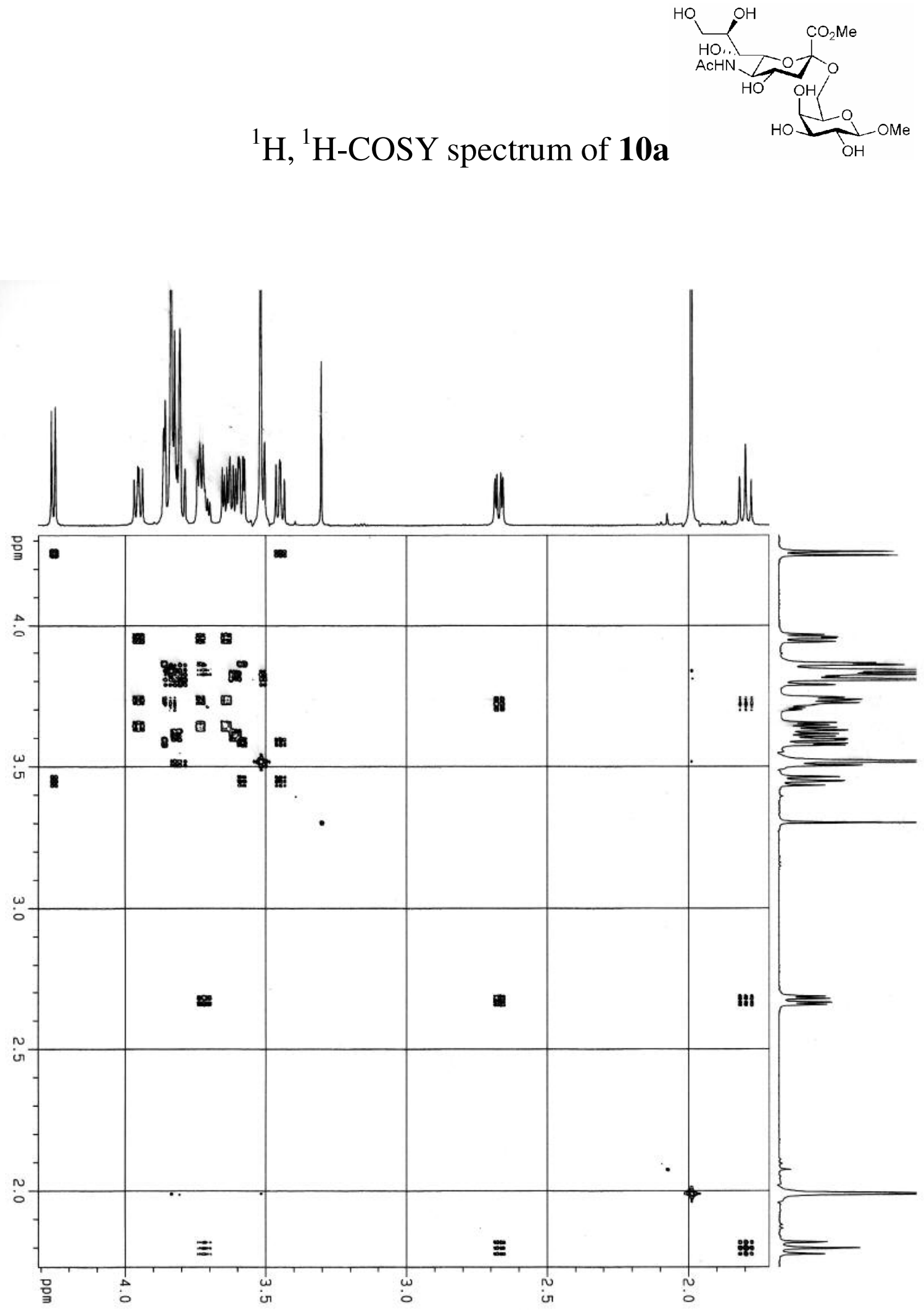

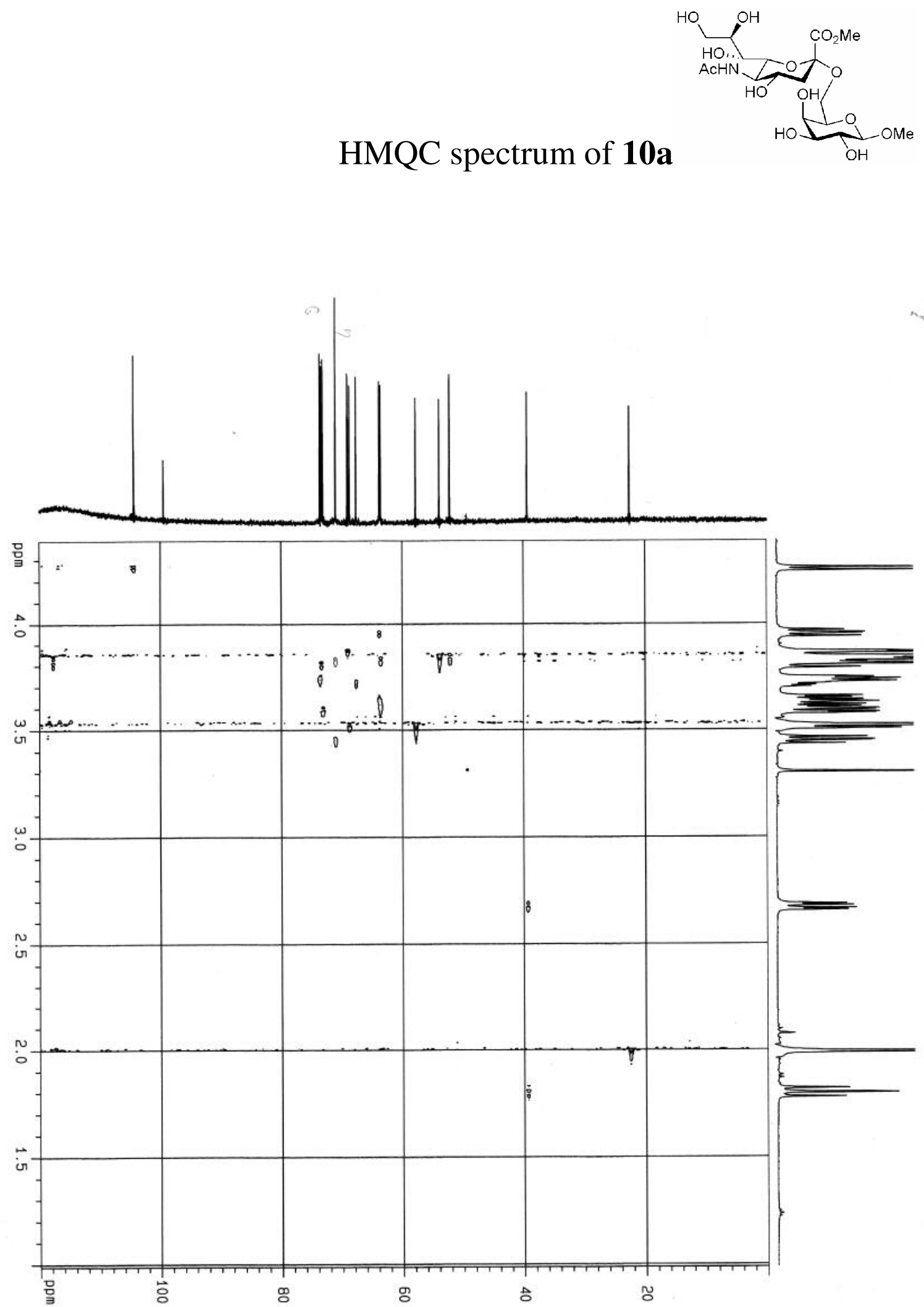

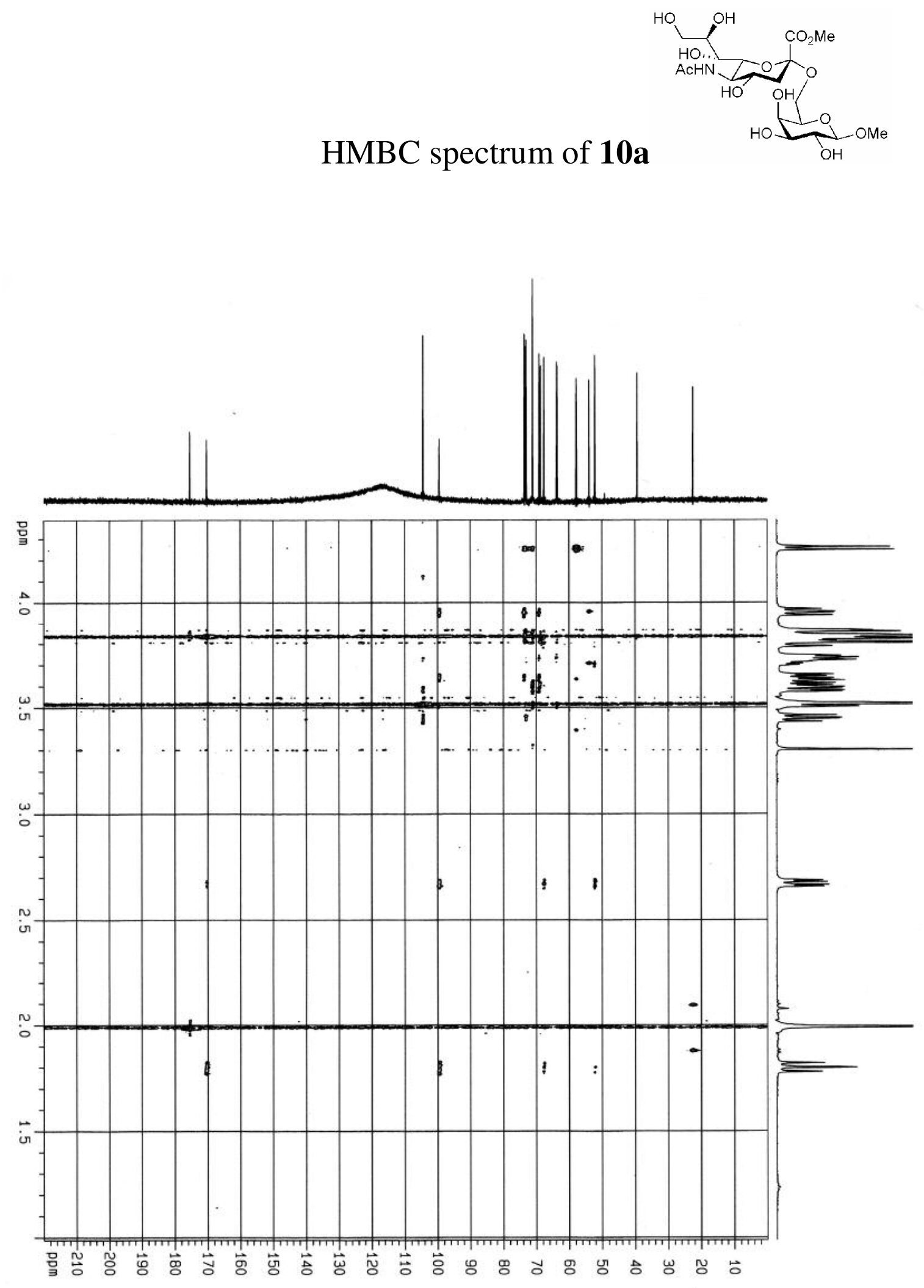

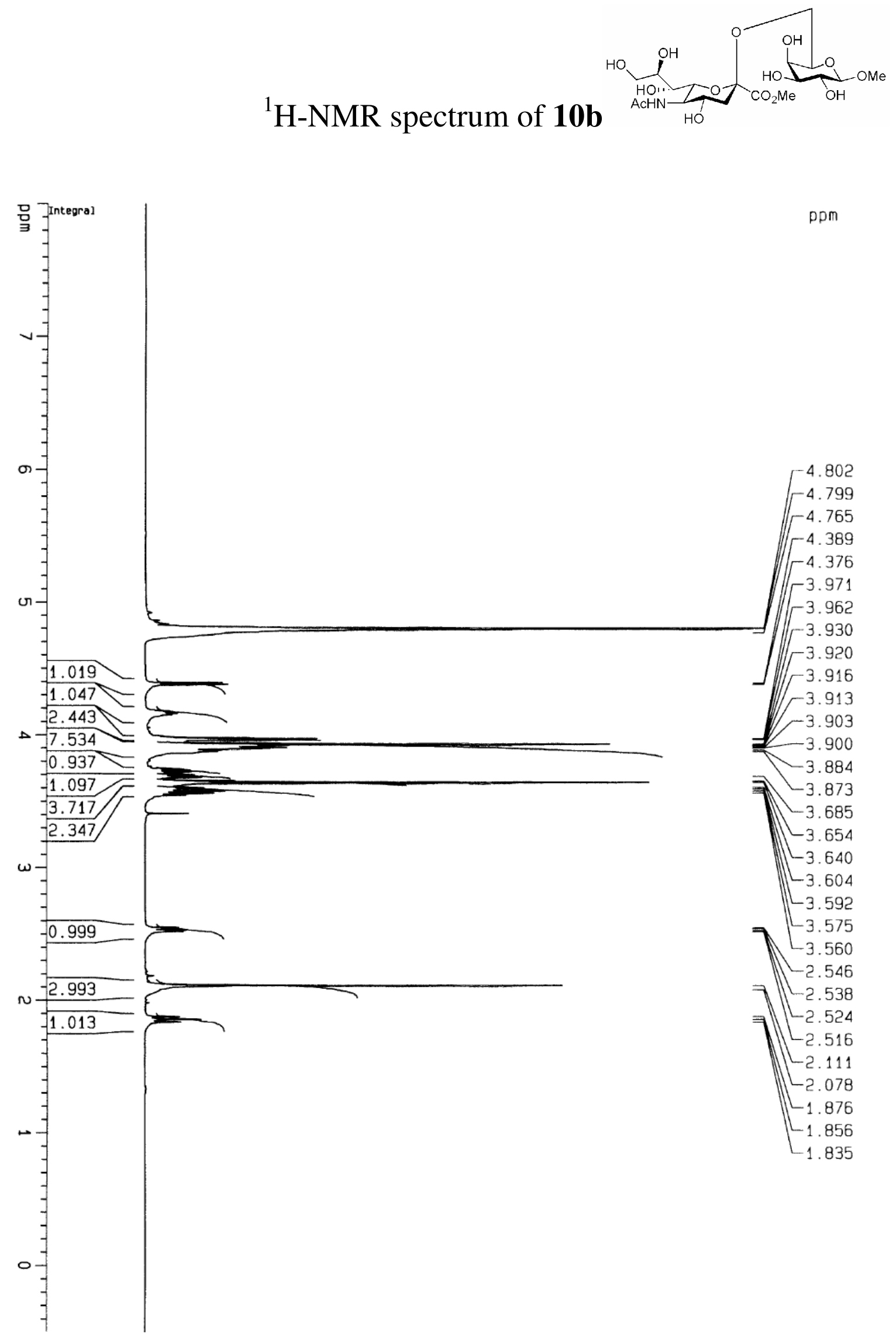
${ }^{13} \mathrm{C}-\mathrm{NMR}$ spectrum of $\mathbf{1 0 b}$
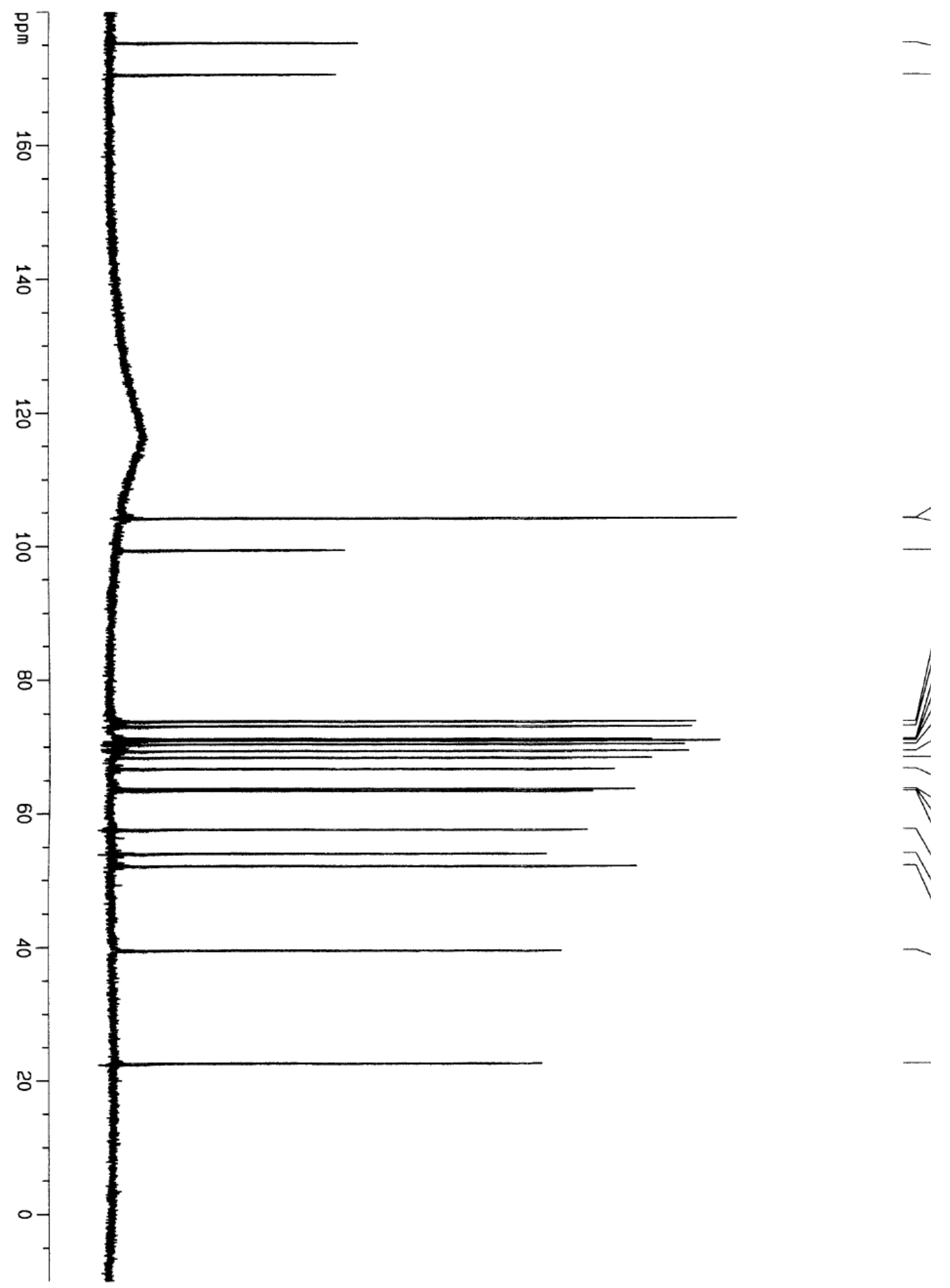


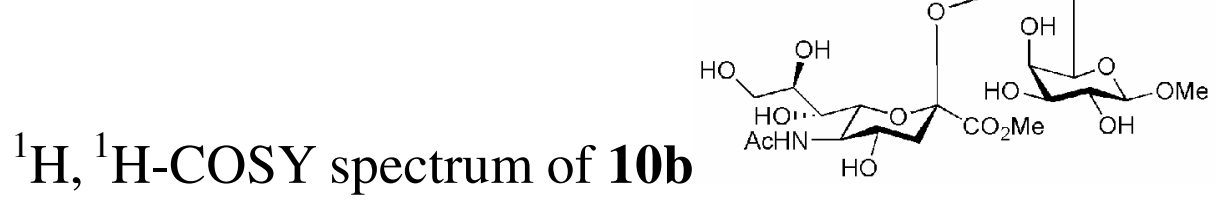

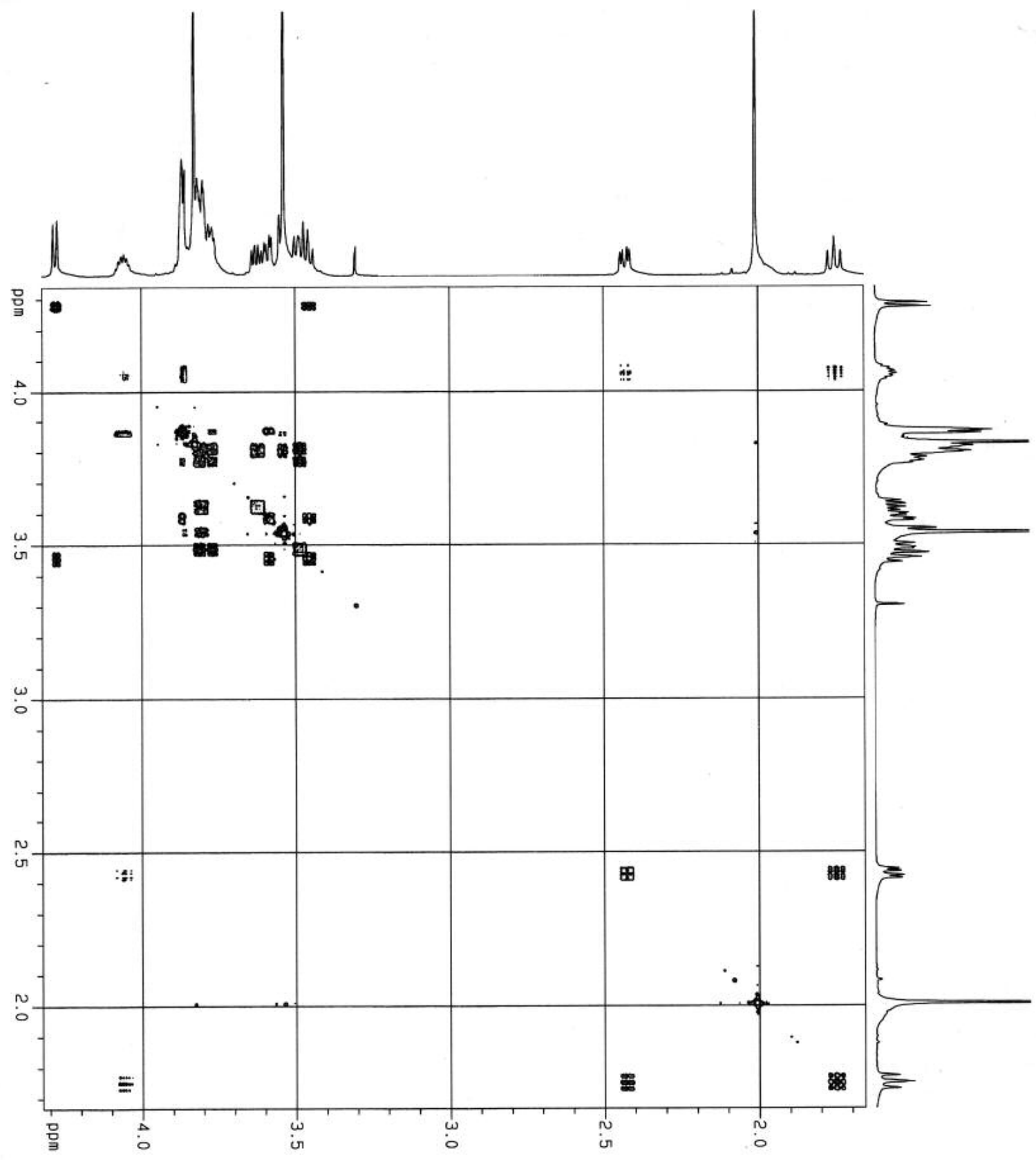



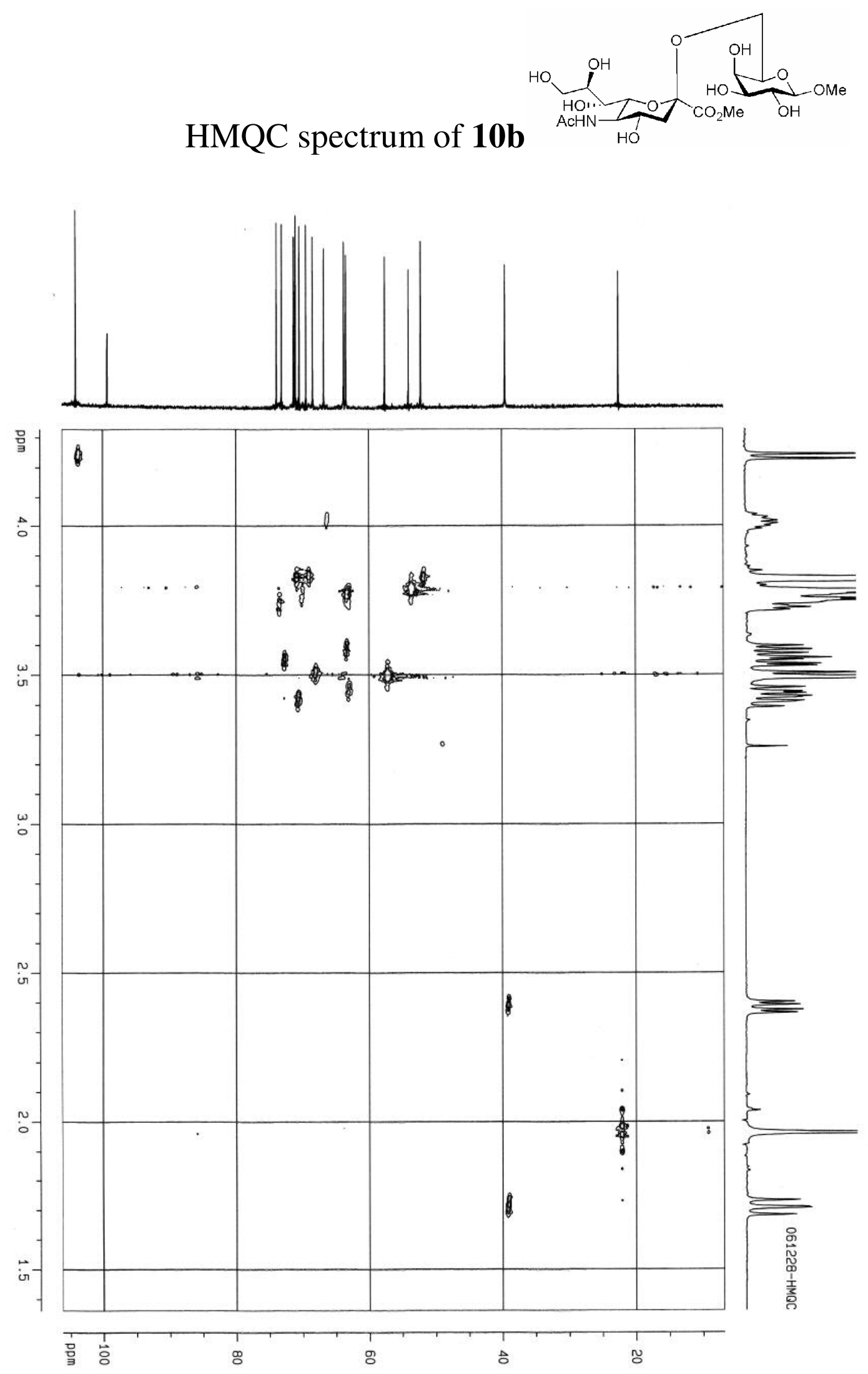

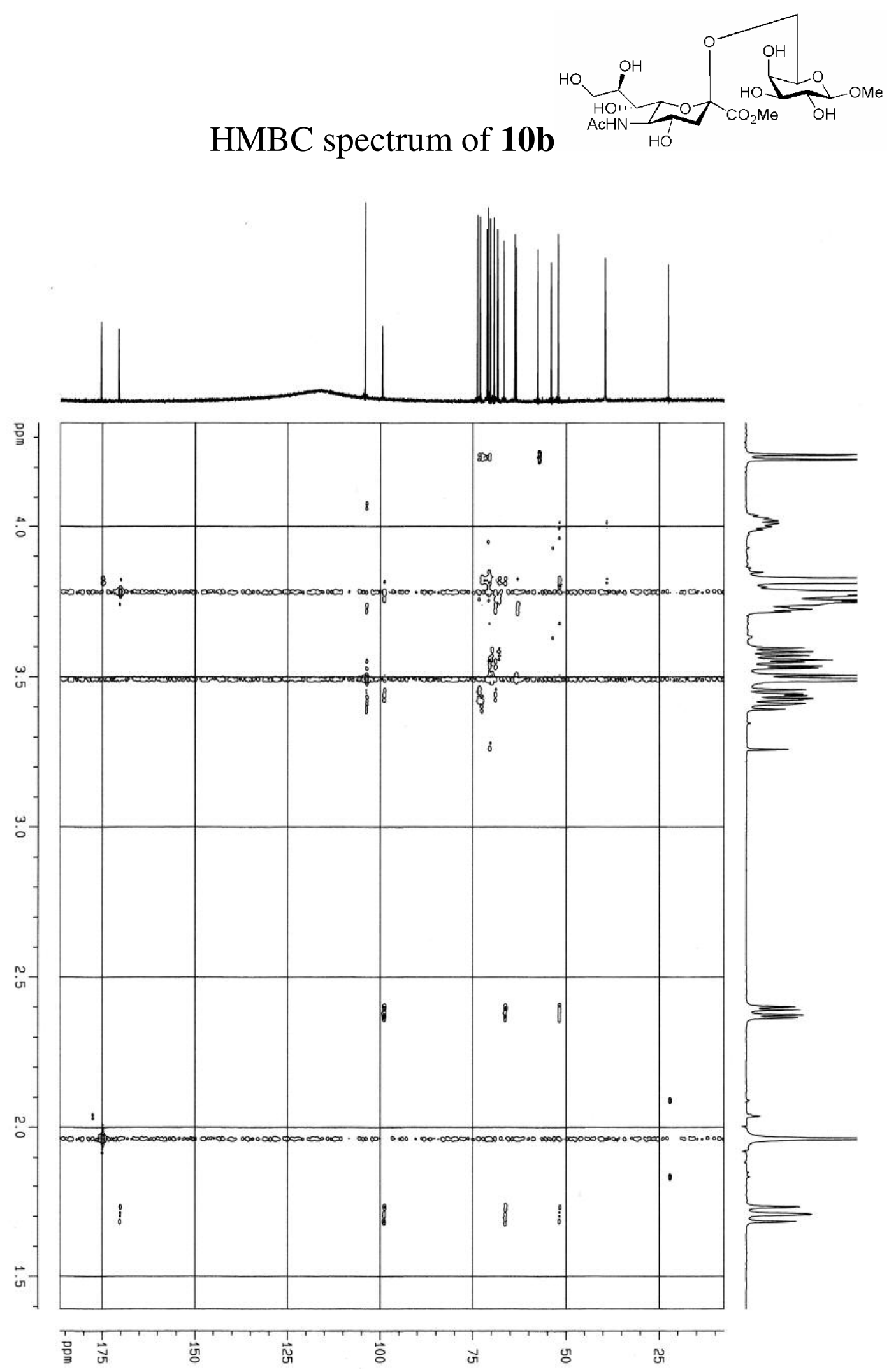